\title{
Induced representations of affine Hecke algebras and canonical bases of quantum groups
}

\author{
Bernard Leclerc, Maxim Nazarov and Jean-Yves Thibon
}

\begin{abstract}
A criterion of irreducibility for induction products of evaluation modules of type $A$ affine Hecke algebras is given. It is derived from multiplicative properties of the canonical basis of a quantum deformation of the Bernstein-Zelevinsky ring.
\end{abstract}

\section{Introduction}

Let $G L_{m}=G L(m, F)$ be the general linear group over a non-Archimedean local field $F$, and $B_{m}$ its subgroup of upper triangular matrices. To each $m$-tuple of complex parameters $\left(s_{1}, \ldots, s_{m}\right)$ is attached a representation $I\left(s_{1}, \ldots, s_{m}\right)$ of $G L_{m}$ in the unramified principal series, which is defined as the right regular representation of $G L_{m}$ on the space of locally constant functions $f: G L_{m} \longrightarrow \mathbf{C}$ satisfying

$$
f(b g)=\chi(b) f(g), \quad\left(b \in B_{m}, g \in G L_{m}\right) .
$$

Here, $\chi$ is the character of $B_{m}$ given on $b=\left(b_{i j}\right)$ by

$$
\chi(b)=\delta(b)^{\frac{1}{2}} \prod_{i=1}^{m}\left|b_{i i}\right|_{F}^{s_{i}},
$$

and

$$
\delta(b)=\prod_{1 \leqslant i<j \leqslant m} \frac{\left|b_{i i}\right|_{F}}{\left|b_{j j}\right|_{F}}=\prod_{i=1}^{m}\left|b_{i i}\right|_{F}^{m+1-2 i}
$$

is the modulus of $B_{m}$. We denote by $\mathcal{C}_{m}$ the category of smooth representations of finite length of $G L_{m}$ whose composition factors are subquotients of $I\left(s_{1}, \ldots, s_{m}\right)$ for some choice of $\left(s_{1}, \ldots, s_{m}\right) \in \mathbf{C}^{m}$.

Let $\mathfrak{o}$ be the ring of integers of $F, \mathfrak{p}$ its maximal ideal, $k=\mathfrak{o} / \mathfrak{p}$ its residue field of cardinality $q$. Let $I_{m}$ be the standard Iwahori subgroup of $G L_{m}$ consisting of those $g=\left(g_{i j}\right) \in G L(m, \mathfrak{o})$ for which $g_{i j} \in \mathfrak{p}$ whenever $i>j$. The space $\widehat{H}_{m}$ of compactly supported complex-valued functions on $G L_{m}$ which are bi-invariant with respect to $I_{m}$ is an algebra under convolution called the Iwahori-Hecke algebra of $G L_{m}$.

By a theorem of Bernstein, Borel, Casselman and Matsumoto (see e.g. Wa ), the category $\mathcal{C}_{m}$ is equivalent to the category $\mathcal{C}\left(\widehat{H}_{m}\right)$ of finite-dimensional complex representations of $\widehat{H}_{m}$. Moreover, one has natural induction functors $\left(M_{1}, M_{2}\right) \mapsto M_{1} \odot M_{2}$ from $\mathcal{C}_{m_{1}} \times \mathcal{C}_{m_{2}}$ to $\mathcal{C}_{m_{1}+m_{2}}$ and from $\mathcal{C}\left(\widehat{H}_{m_{1}}\right) \times \mathcal{C}\left(\widehat{H}_{m_{2}}\right)$ to $\mathcal{C}\left(\widehat{H}_{m_{1}+m_{2}}\right)$ which correspond to each other via 
the equivalences of categories. In this paper we want to study under which conditions induction products $L_{1} \odot L_{2}$ of irreducible objects of these categories are irreducible.

Following Bernstein and Zelevinsky, one introduces

$$
\mathcal{R}=\bigoplus_{m \geqslant 0} \mathcal{R}_{m}
$$

where for $m \geqslant 1, \mathcal{R}_{m}$ denotes the complexified Grothendieck group of $\mathcal{C}_{m}$ (or $\mathcal{C}\left(\widehat{H}_{m}\right)$ ), and $\mathcal{R}_{0}:=$ C. The induction and restriction functors make $\mathcal{R}$ into a Hopf algebra which was explicitely described by Zelevinsky [Ze1. For $s \in \mathbf{C}$, let $\mathcal{R}_{m}(s)$ be the subgroup of $\mathcal{R}_{m}$ generated by the classes of the subquotients of all representations $I\left(s_{1}, \ldots, s_{m}\right)$ for which $s_{1}, \ldots, s_{m} \in s+\mathbf{Z}$, and let

$$
\mathcal{R}(s)=\bigoplus_{m \geqslant 0} \mathcal{R}_{m}(s)
$$

Note that, since $|x|_{F}^{s}=q^{-s \operatorname{val}_{F}(x)}$ depends only on $s$ modulo $(2 \pi i / \log q) \mathbf{Z}$, the group $\mathcal{R}(s)$ depends only on $s$ modulo $\Omega=\mathbf{Z} \oplus(2 \pi i / \log q) \mathbf{Z}$. Now ([Ze1, 8.7), $\mathcal{R}(s)$ is a subalgebra of $\mathcal{R}$, the $\mathcal{R}(s)$ are all isomorphic to each other, and $\mathcal{R}=\bigotimes \mathcal{R}(s)$ where $s$ runs over the elliptic curve $\mathbf{C} / \Omega$. So it is enough to describe $R:=\mathcal{R}(0)$.

It turns out that $R$ is isomorphic, as a Hopf algebra, to the algebra of polynomials in the coordinate functions of the group $N_{\infty}$ of upper triangular unipotent $\mathbf{Z} \times \mathbf{Z}$-matrices with finitely many non-zero entries off the main diagonal. As such, $R$ has a natural quantum deformation $R_{v}$ (in the sense of Drinfeld and Jimbo). By results of Kashiwara and Lusztig, $R_{v}$ is endowed with a canonical basis $\mathcal{B}_{v}$ which specializes when $v \mapsto 1$ to a canonical basis $\mathcal{B}$ of $R$. Our approach to the representation theory of $G L_{m}$ and $\widehat{H}_{m}$ is based on the following crucial fact:

The canonical basis $\mathcal{B}$ coincides with the basis of $R$ consisting of the classes of irreducible representations.

This theorem follows by comparing the p-adic analogue of the Kazhdan-Lusztig formula, conjectured by Zelevinsky [Ze2 and proved by Ginzburg [CG], with the geometrical description by Lusztig of the canonical basis [Lu1. The dual version of this theorem is proved in $\underline{\mathbf{A r}}$.

Here $\mathcal{B}_{v}$ is a dual canonical basis in the sense of Lusztig, or an upper global basis in the sense of Kashiwara. A distinguished subset of $\mathcal{B}_{v}$ consists of the quantum flag minors [BZ1, LZ]. The quantum flag minors of degree $m$ correspond to a special class of irreducible $\widehat{H}_{m}$-modules, called the evaluation modules. They are obtained by lifting the simple modules of the finite-dimensional Hecke algebra $H_{m}$ via the evaluation maps (see below $\$ 2.4)$. In the equivalence of categories $\mathcal{C}\left(\widehat{H}_{m}\right) \simeq \mathcal{C}_{m}$, these modules correspond in turn to a large class of irreducible representations of $G L_{m}$, namely those parametrized, up to a shift by an arbitrary $z \in \mathbf{C}^{*}$, by Zelevinsky's multi-segments of the form

$$
\mathbf{m}=\sum_{k=1}^{r}\left[i+k-1, j_{k}\right],
$$

where $i, j_{1}, \ldots, j_{k}$ are integers, and $i<j_{1}<j_{2}<\cdots<j_{r}$.

The theorem above implies that multiplying vectors of $\mathcal{B}$ is the same as taking induction products of irreducible modules in $R$, and therefore we are led to the problem of understanding which products of elements of $\mathcal{B}$ belong to $\mathcal{B}$. The multiplicative properties 
of the dual canonical basis have been studied by Berenstein and Zelevinsky BZ1]. They conjectured that the product of two vectors of $\mathcal{B}_{v}$ belongs to $\mathcal{B}_{v}$ up to a power of $v$, if and only if these vectors commute up to a power of $v$. We will prove this conjecture in the special case of the quantum flag minors, for which we have an explicit criterion of $v$-commutativity [LZ. This will give a criterion for the irreducibility of induction products of evaluation modules. Namely, for $z \in \mathbf{C}^{*}$ and $\alpha$ a partition of $m$, let $S_{\alpha}(z)$ denote the $\widehat{H}_{m}$-module obtained by evaluation at $z$ of the simple $H_{m}$-module $S_{\alpha}$ attached to a partition $\alpha$ of $m \geqslant 1$. Similarly, let $S_{\beta}(w)$ be an evaluation module of $\widehat{H}_{n}(z)$ for some $n \geqslant 1$. Since the induction product $S_{\alpha}(z) \odot S_{\beta}(w)$ is simple if $z / w \notin q^{\mathbf{Z}}$ [Ze1], we can assume that $z / w \in q^{\mathbf{Z}}$. Our main result is

Theorem 1 Let $z / w=q^{c}$ for some $c \in \mathbf{Z}$. Associate to the partitions $\alpha=\left(\alpha_{1}, \ldots, \alpha_{r}\right)$ and $\beta=\left(\beta_{1}, \ldots, \beta_{s}\right)$ the following subsets of $\mathbf{Z}$ :

$$
\mathcal{I}=\mathbf{Z}_{\leqslant c-r} \cup\left\{c-r+1+\alpha_{r}, \ldots, c+\alpha_{1}\right\}, \quad \mathcal{J}=\mathbf{Z}_{\leqslant-s} \cup\left\{-s+1+\beta_{s}, \ldots, \beta_{1}\right\} .
$$

(i) Suppose c $>0$. Then, the product $S_{\alpha}(z) \odot S_{\beta}(w)$ is not simple if and only if there exist $i, j, k \in \mathbf{Z}$ such that $i, k \in \mathcal{I} \backslash \mathcal{J}, j \in \mathcal{J} \backslash \mathcal{I}$ and $i<j<k$.

(ii) Suppose $c<0$. Then, the product $S_{\alpha}(z) \odot S_{\beta}(w)$ is not simple if and only if there exist $i, j, k \in \mathbf{Z}$ such that $i, k \in \mathcal{J} \backslash \mathcal{I}, j \in \mathcal{I} \backslash \mathcal{J}$ and $i<j<k$.

(iii) Suppose $c=0$. Then, the product $S_{\alpha}(z) \odot S_{\beta}(w)$ is not simple if and only if there exist $i, j, k, l \in \mathbf{Z}$ such that $i, k \in \mathcal{I} \backslash \mathcal{J}, j, l \in \mathcal{J} \backslash \mathcal{I}$ and either $i<j<k<l$ or $j<i<l<k$.

Note that $S_{\alpha}(z) \odot S_{\beta}(w)$ is simple if and only if $S_{\beta}(w) \odot S_{\alpha}(z)$ is simple. Hence, the statements (i) and (ii) of our Theorem 1 1 are in fact equivalent. Using an argument of Kitanine, Maillet and Terras [KMT] IMT] (see also [NT2]), we deduce from Theorem 1 an irreducibility criterion for products of any number of evaluation modules, by translating to representations of affine quantum groups.

Theorem 2 Let $z_{1}, \ldots, z_{r}$ be non-zero complex numbers and $\alpha^{(1)}, \ldots, \alpha^{(r)}$ be partitions of some positive integers. The product $S_{\alpha^{(1)}}\left(z_{1}\right) \odot \cdots \odot S_{\alpha^{(r)}}\left(z_{r}\right)$ is simple if and only if the products $S_{\alpha^{(k)}}\left(z_{k}\right) \odot S_{\alpha^{(l)}}\left(z_{l}\right)$ are simple for all $1 \leqslant k<l \leqslant r$.

For partitions $\alpha^{(1)}, \ldots, \alpha^{(r)}$ of certain special types, the irreducibility criterion for the induced module $S_{\alpha^{(1)}}\left(z_{1}\right) \odot \cdots \odot S_{\alpha^{(r)}}\left(z_{r}\right)$ has been known. When $\alpha^{(k)}=(1)$ for every $k=1, \ldots, r$ the induced module belongs to the principal series, and the irreducibility criterion has been known for a long time (in Kat this criterion was given for the affine Hecke algebras corresponding to arbitrary root systems). When each of the partitions $\alpha^{(1)}, \ldots, \alpha^{(r)}$ consists of one part only, the induced module belongs to the generalized principal series, and the irreducibility criterion was given by Zelevinsky [Ze1] in terms of segments.

We note that Reineke has also proven a partial case of the Berenstein-Zelevinsky conjecture, namely when one of the two vectors is a "small" quantum minor $\mathbf{R e}$.

Our paper is organized as follows. In Section 2 we recall the classification of the irreducible finite-dimensional representations of the affine Hecke algebras, the description of the Bernstein-Zelevinsky algebra $R$, and the formula for the composition multiplicities of the standard induced modules. In Section 3, we review the definitions of the quantum algebras $U_{v}^{+}=U_{v}^{+}\left(\mathfrak{s} l_{\infty}\right)$ and $R_{v}$ and of their canonical bases. In Section 4 , we describe a simple algorithm for calculating the dual canonical basis of $U_{v}^{+}$. In Section 5 , we recall the Berenstein-Zelevinsky conjecture about multiplicative properties of this basis, as well 
as the criterion of quasi-commutativity of [LZ for quantum flag minors. In Section 6] we state our results about the Berenstein-Zelevinsky conjecture for flag minors, and we prove most of them in Section [7. Finally in Section 8 we return to evaluation modules of affine Hecke algebras and derive our main results.

\section{Representations of affine Hecke algebras}

2.1 By a theorem of Bernstein, the Iwahori-Hecke algebra $\widehat{H}_{m}=\widehat{H}_{m}(q)$ of $G L_{m}$ has the following presentation. It is the associative $\mathbf{C}$-algebra with invertible generators $y_{1}, \ldots, y_{m}$ and $T_{1}, \ldots, T_{m-1}$ subject to the relations

$$
\begin{array}{ll}
T_{i} T_{i+1} T_{i}=T_{i+1} T_{i} T_{i+1}, & 1 \leqslant i \leqslant m-2, \\
T_{i} T_{j}=T_{j} T_{i}, & |i-j|>1, \\
\left(T_{i}-q\right)\left(T_{i}+1\right)=0, & 1 \leqslant i \leqslant m-1, \\
y_{i} y_{j}=y_{j} y_{i}, & 1 \leqslant i, j \leqslant m, \\
y_{j} T_{i}=T_{i} y_{j}, & j \neq i, i+1, \\
T_{i} y_{i} T_{i}=q y_{i+1}, & 1 \leqslant i \leqslant m-1 .
\end{array}
$$

More generally, one can define for any $t \in \mathbf{C}^{*}$ an algebra $\widehat{H}_{m}(t)$ by replacing $q$ by $t$ in this presentation. It is known that the representation theory of $\widehat{H}_{m}(t)$ is the same for all parameters $t$ which are not roots of unity, so from now on we only assume that $t$ is a complex number of infinite multiplicative order and we write $\widehat{H}_{m}$ in place of $\widehat{H}_{m}(t)$. In particular, our proofs of Theorem 1 and Theorem 2 will be valid for any such parameter $t$. Therefore, when we refer below to Theorem [1] and Theorem [2, it should be understood that the parameter $q$ of Section 1 is replaced by such a generic complex number $t$.

2.2 The classification of irreducible finite-dimensional representations of $\widehat{H}_{m}$ was obtained by Zelevinsky [Ze1] (in the case $t=q$; for the general case see for instance Ro2 ). Let $\mathcal{A}_{m}$ denote the commutative subalgebra of $\widehat{H}_{m}$ generated by the elements $y_{i}^{ \pm}(1 \leqslant i \leqslant m)$. As explained in the introduction, we may restrict ourselves to the category of finite-dimensional representations in which the generators $y_{i}$ of $\mathcal{A}_{m}$ have all their eigenvalues in $t^{\mathbf{Z}}$. Let $\mathcal{C}_{m}^{\mathbf{Z}}$ denote this category. The parametrization of the simple modules of $\mathcal{C}_{m}^{\mathbf{Z}}$ is in terms of combinatorial objects called multi-segments. It is obtained as follows. For $\mu=\left(\mu_{1}, \ldots, \mu_{r}\right)$ a composition of $m$, set

$$
D(\mu)=\left\{\mu_{1}+\cdots+\mu_{k}, 1 \leqslant k \leqslant r-1\right\}=\left\{d_{1}, \ldots, d_{r-1}\right\}
$$

and denote by $\widehat{H}_{\mu}$ the subalgebra of $\widehat{H}_{m}$ generated by $\mathcal{A}_{m}$ and $\left\{T_{i} \mid i \notin D(\mu)\right\}$. For $\mathbf{a}=\left(a_{1}, \ldots, a_{r}\right) \in \mathbf{Z}^{r}$, let $\mathbf{C}_{\mu, \mathbf{a}}$ be the 1-dimensional representation of $\widehat{H}_{\mu}$ defined by $T_{i} \mapsto t, i \notin D(\mu)$, and $y_{d_{i-1}+1} \mapsto t^{a_{i}}, i=1, \ldots, r$, where $d_{0}=0$. (Observe that because of the defining relations of $\widehat{H}_{m}(t)$ the action of the other generators $y_{j}$ on $\mathbf{C}_{\mu, \mathbf{a}}$ is then given by $y_{d_{i-1}+k} \mapsto t^{a_{i}+k-1}, k=1, \ldots, \mu_{i}$.) We denote by $M_{\mu, \mathbf{a}}$ the induced $\widehat{H}_{m}$-module

$$
M_{\mu, \mathbf{a}}=\widehat{H}_{m} \otimes_{\widehat{H}_{\mu}} \mathbf{C}_{\mu, \mathbf{a}} .
$$

The representations $M_{\mu, \mathbf{a}}$ belong to the so-called generalized principal series. In particular, when $\mu=(1, \ldots, 1)=\left(1^{m}\right)$ and $\mathbf{a}=\left(a_{1}, \ldots, a_{m}\right)$, we get a principal series representation of $\widehat{H}_{m}$. 
Let $R_{m}$ be the complexified Grothendieck group of the category $\mathcal{C}_{m}^{\mathbf{Z}}$. When $(\nu, \mathbf{b})$ is a permutation of $(\mu, \mathbf{a})$, i.e. $\nu_{i}=\mu_{\sigma(i)}$ and $b_{i}=a_{\sigma(i)}$ for some $\sigma \in \mathfrak{S}_{r}$, the induced modules $M_{\mu, \mathbf{a}}$ and $M_{\nu, \mathbf{b}}$ are in general non isomorphic, but their classes in $R_{m}$ are equal [Ze1, that is, the modules $M_{\mu, \mathbf{a}}$ and $M_{\nu, \mathbf{b}}$ have the same composition factors with identical multiplicities.

The equivalence class of $(\mu, \mathbf{a})$ considered up to permutations is nothing but a multisegment. Here, by a segment we mean an interval $[i, j]$ in $\mathbf{Z}$, and we call multi-segment a formal finite unordered sum $\mathbf{m}=\sum_{i \leqslant j} m_{i j}[i, j]$. The integer $m_{i j}$ is the multiplicity of the segment $[i, j]$ in $\mathbf{m}$. When $i=j$ we sometimes write $[i]$ instead of $[i, i]$. We define the degree of $\mathbf{m}$ by $\operatorname{deg}(\mathbf{m})=\sum_{i \leqslant j} m_{i j}(j-i+1)$. Now, by attaching to $(\mu, \mathbf{a})$ the multi-segment

$$
\mathbf{m}=\sum_{i=1}^{r}\left[a_{i}, a_{i}+\mu_{i}-1\right],
$$

we obviously get a one-to-one correspondence between the classes of pairs $(\mu, \mathbf{a})$ as above and the multi-segments $\mathbf{m}$ of degree $m$. Thus we can write unambiguously $\left[M_{\mathbf{m}}\right]$ for the class of $M_{\mu, \mathbf{a}}$ in $R_{m}$. The set of segments and the set of multi-segments will be denoted respectively by $\mathcal{S}$ and $\mathcal{M}$.

Zelevinsky has introduced the following partial order on $\mathcal{M}$ [Ze1, Ze2]. We say that two segments $\mathbf{s}$ and $\mathbf{s}^{\prime}$ are linked when $\mathbf{t}=\mathbf{s} \cup \mathbf{s}^{\prime}$ is a segment and $\mathbf{t}$ is different from $\mathbf{s}$ and $\mathbf{s}^{\prime}$. Let $\mathbf{m}$ and $\mathbf{n}$ be two distinct multi-segments. We write $\mathbf{m} \rightarrow \mathbf{n}$ if the multi-segment $\mathbf{n}$ can be obtained from $\mathbf{m}$ by replacing a pair $\mathbf{s}, \mathbf{s}^{\prime}$ of linked segments of $\mathbf{m}$ by the pair $\mathbf{t}=\mathbf{s} \cup \mathbf{s}^{\prime}, \mathbf{t}^{\prime}=\mathbf{s} \cap \mathbf{s}^{\prime}$, where $\mathbf{t}^{\prime}$ is allowed to be empty. More generally we write $\mathbf{m} \triangleleft \mathbf{n}$ if there exists a sequence of multi-segments $\mathbf{n}_{1}, \ldots, \mathbf{n}_{k}$ such that $\mathbf{m} \rightarrow \mathbf{n}_{1} \rightarrow \cdots \rightarrow \mathbf{n}_{k} \rightarrow \mathbf{n}$. It is known [Ze1, Ze2 that $M_{\mathbf{m}}$ is irreducible if and only if $\mathbf{m}$ is maximal for $\unlhd$, and if this is not the case, there is a unique simple module whose class occurs in the expansion of $\left[M_{\mathbf{m}}\right]$ but does not occur in any $\left[M_{\mathbf{n}}\right]$ with $\mathbf{m} \triangleleft \mathbf{n}$. Let $L_{\mathbf{m}}$ denote this simple module (if $\mathbf{m}$ is maximal, $L_{\mathbf{m}}=M_{\mathbf{m}}$ ). Then the $L_{\mathbf{m}}$ are pairwise non isomorphic and all simple modules of $\mathcal{C}_{m}^{\mathbf{Z}}$ are of this type for some multi-segment $\mathbf{m}$ of degree $m$.

Example 3 If $\widehat{H}_{m}=\widehat{H}_{m}(q)$ is the Iwahori-Hecke algebra of $G L_{m}$, then each finitedimensional representation $M$ of $\widehat{H}_{m}$ can be identified with the subspace of $I_{m}$-fixed vectors in a smooth representation of finite length $V_{M}$ of $G L_{m}$. In this correspondence the principal series representation $M_{\left(1^{m}\right), \mathbf{a}}$ of $\widehat{H}_{m}$ is sent to the principal series representation $I\left(-a_{1}, \ldots,-a_{m}\right)$ of $G L_{m}$.

For an irreducible representation $M=L_{\mathbf{m}}$ of $\widehat{H}_{m}$, let $V_{\mathbf{m}}=V_{M}$ denote the corresponding irreducible representation of $G L_{m}$. For instance, consider for $i \in \mathbf{Z}$ the multi-segments

$$
\mathbf{m}(i)=[i, i+m-1], \quad \mathbf{n}(i)=[i]+[i+1]+\cdots+[i+m-1] .
$$

Then, $L_{\mathbf{m}(i)}$ is the 1-dimensional $\widehat{H}_{m}$-module in which all $T_{i}$ 's act by multiplication by $t$, and $y_{1}$ by multiplication by $t^{i}$. On the other hand, $L_{\mathbf{n}(i)}$ is the 1-dimensional $\widehat{H}_{m^{-}}$ module in which all $T_{i}$ 's act by multiplication by -1 , and $y_{1}$ by multiplication by $t^{i+m-1}$. Correspondingly, $V_{\mathbf{m}(i)}$ is the 1-dimensional representation attached to the character

$$
g \mapsto|\operatorname{det} g|_{F}^{-i-\frac{m-1}{2}}
$$

of $G L_{m}$, and $V_{\mathbf{n}(i)}=V_{\mathbf{m}(i)} \otimes \mathrm{St}$, where St denotes the special (or Steinberg) representation, that is, the unique irreducible quotient of the right regular representation of $G L_{m}$ on the space of locally constant functions on $B_{m} \backslash G L_{m}$. 


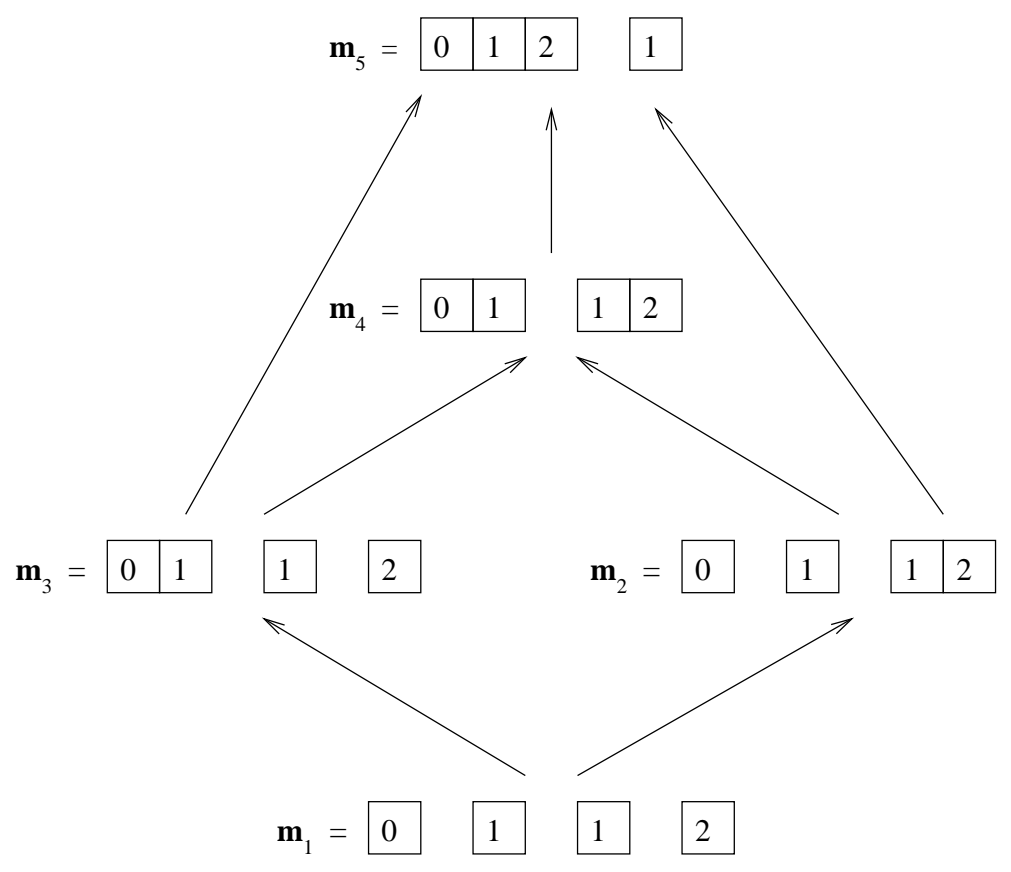

Figure 1: The multi-segments larger than $[0]+2[1]+[2]$

Example 4 Let $\mathbf{m}_{1}=[0]+2[1]+[2]$. This a minimal element for $\unlhd$. The multi-segments larger than $\mathbf{m}_{1}$ for $\unlhd$ are

$$
\mathbf{m}_{2}=[0]+[1]+[1,2], \mathbf{m}_{3}=[0,1]+[1]+[2], \mathbf{m}_{4}=[0,1]+[1,2], \mathbf{m}_{5}=[1]+[0,2],
$$

and the poset structure is illustrated in Figure 1, One has ([Ze1], 11.4):

$$
\left\{\begin{array}{l}
{\left[M_{\mathbf{m}_{5}}\right]=\left[L_{\mathbf{m}_{5}}\right]} \\
{\left[M_{\mathbf{m}_{4}}\right]=\left[L_{\mathbf{m}_{4}}\right]+\left[L_{\mathbf{m}_{5}}\right]} \\
{\left[M_{\mathbf{m}_{3}}\right]=\left[L_{\mathbf{m}_{3}}\right]+\left[L_{\mathbf{m}_{4}}\right]+\left[L_{\mathbf{m}_{5}}\right]} \\
{\left[M_{\mathbf{m}_{2}}\right]=\left[L_{\mathbf{m}_{2}}\right]+\left[L_{\mathbf{m}_{4}}\right]+\left[L_{\mathbf{m}_{5}}\right]} \\
{\left[M_{\mathbf{m}_{1}}\right]=\left[L_{\mathbf{m}_{1}}\right]+\left[L_{\mathbf{m}_{2}}\right]+\left[L_{\mathbf{m}_{3}}\right]+2\left[L_{\mathbf{m}_{4}}\right]+\left[L_{\mathbf{m}_{5}}\right],}
\end{array}\right.
$$

which, by solving the equations, gives:

$$
\left\{\begin{array}{l}
{\left[L_{\mathbf{m}_{5}}\right]=\left[M_{\mathbf{m}_{5}}\right]} \\
{\left[L_{\mathbf{m}_{4}}\right]=\left[M_{\mathbf{m}_{4}}\right]-\left[M_{\mathbf{m}_{5}}\right]} \\
{\left[L_{\mathbf{m}_{3}}\right]=\left[M_{\mathbf{m}_{3}}\right]-\left[M_{\mathbf{m}_{4}}\right]} \\
{\left[L_{\mathbf{m}_{2}}\right]=\left[M_{\mathbf{m}_{2}}\right]-\left[M_{\mathbf{m}_{4}}\right]} \\
{\left[L_{\mathbf{m}_{1}}\right]=\left[M_{\mathbf{m}_{1}}\right]-\left[M_{\mathbf{m}_{2}}\right]-\left[M_{\mathbf{m}_{3}}\right]+\left[M_{\mathbf{m}_{5}}\right] .}
\end{array}\right.
$$

2.3 The composition multiplicities of the induced modules $M_{\mathbf{m}}$ are given by the socalled $p$-adic analogue of the Kazhdan-Lusztig conjecture formulated by Zelevinsky [Ze2 and proved by Ginzburg [CG], Theorem 8.6.23. (See also $\mathbf{S u}$ ] for another proof in the case of the degenerate affine Hecke algebra.) Let us recall this result. 
Fix a multi-segment $\mathbf{m}=\sum_{i \leqslant j} m_{i j}[i, j]$, and set $d_{k}=\sum_{i \leqslant k \leqslant j} m_{i j}(k \in \mathbf{Z})$. Clearly, only a finite number of $d_{k}$ are non-zero. We call $d=\left(d_{k}\right)_{k \in \mathbf{Z}}$ the weight of $\mathbf{m}$ and write $\mathrm{wt}(\mathbf{m})=d$.

Let $V=\bigoplus_{k \in \mathbf{Z}} V_{k}$ be a $\mathbf{Z}$-graded vector space over $\mathbf{C}$ with $\operatorname{dim} V_{k}=d_{k}$. Let $E_{V}$ be the set of endomorphisms $x$ of $V$ of degree -1 , i.e. such that $x V_{k} \subset V_{k-1}$. (Equivalently, if $\Gamma$ denotes the quiver of type $A_{\infty}$ with orientation $k \longrightarrow k-1$ for all $k \in \mathbf{Z}$, then $E_{V}$ is the set of representations of $\Gamma$ over $\mathbf{C}$ whose graded dimension is $d$.)

The group $G_{V}=\prod_{k \in \mathbf{Z}} G L\left(V_{k}\right)$ acts on $E_{V}$ by conjugation. (The orbits of this action are precisely the isomorphism classes of representations of $\Gamma$ with dimension $d$.) Note that all $x \in E_{V}$ are nilpotent and admit a Jordan basis $b$ consisting of homogeneous elements. Define the graded Jordan type of $x \in E_{V}$ as the multi-segment $\mathbf{n}=\sum_{i \leqslant j} n_{i j}[i, j]$, where $n_{i j}$ is the number of Jordan cells of $b$ starting in $V_{j}$ and ending in $V_{i}$. It is easy to see that the $G_{V}$-orbit of $x$ consists of those elements $y$ having the same graded Jordan type as $x$. Hence, the $G_{V}$-orbits $O_{\mathbf{n}}$ in $E_{V}$ are parametrized by the multi-segments $\mathbf{n}$ of weight $d$.

Zelevinsky has shown [Ze2 that the closure of $O_{\mathbf{n}}$ decomposes as $\bar{O}_{\mathbf{n}}=\coprod_{\mathbf{m} \unlhd \mathbf{n}} O_{\mathbf{m}}$, so that, $\mathbf{m} \unlhd \mathbf{n}$ if and only if $O_{\mathbf{m}} \subset \bar{O}_{\mathbf{n}}$. Let $\mathcal{H}^{i}\left(\bar{O}_{\mathbf{n}}\right)_{\mathbf{m}}$ denote the stalk at a point $x \in O_{\mathbf{m}}$ of the $i$ th intersection cohomology sheaf of the variety $\bar{O}_{\mathbf{n}}$.

Theorem 5 ([Ze2], $[\mathbf{C G}]$ ) The composition multiplicity of the simple module $L_{\mathbf{n}}$ in the induced module $M_{\mathbf{m}}$ is equal to

$$
K_{\mathbf{m n}}=\sum_{i \geqslant 0} \operatorname{dim} \mathcal{H}^{i}\left(\bar{O}_{\mathbf{n}}\right)_{\mathbf{m}} .
$$

In $\left[\mathbf{Z e 3}\right.$, Zelevinsky has further shown that the varietes $\bar{O}_{\mathbf{n}}$ are locally isomorphic to some Schubert varieties of type $A_{m-1}$, where $m=\operatorname{deg}(\mathbf{n})$. Hence $K_{\mathbf{m n}}$ is the value at $v=1$ of a certain Kazhdan-Lusztig polynomial $K_{\mathbf{m n}}(v)$ for the symmetric group $\mathfrak{S}_{m}$.

2.4 Let $H_{m}$ denote the subalgebra of $\widehat{H}_{m}$ generated by the elements $T_{i}(1 \leqslant i \leqslant m-1)$. This is a Iwahori-Hecke algebra of type $A_{m-1}$. The irreducible $H_{m}$-modules are known to be parametrized by the partitions $\alpha$ of $m$. Let us denote them by $S_{\alpha}$. In particular, $S_{(m)}$ and $S_{\left(1^{m}\right)}$ stand for the one-dimensional $H_{m}$-modules associated to the characters $T_{i} \mapsto t$ and $T_{i} \mapsto-1$, respectively.

For $z \in \mathbf{C}^{*}$ there exists a unique surjective homomorphism $\tau_{z}: \widehat{H}_{m} \rightarrow H_{m}$ such that

$$
\tau_{z}\left(T_{i}\right)=T_{i} \quad(1 \leqslant i \leqslant m-1), \quad \tau_{z}\left(y_{1}\right)=z .
$$

This is called the evaluation of $\widehat{H}_{m}$ at $z$. By pulling back the $H_{m}$-module $S_{\alpha}$ via $\tau_{z}$, one gets an irreducible representation of $\widehat{H}_{m}$ denoted by $S_{\alpha}(z)$ and called an evaluation module. It belongs to $\mathcal{C}_{m}^{\mathbf{Z}}$ when $z=t^{a}$ for some $a \in \mathbf{Z}$. Consider the Young diagram $Y_{\alpha}(a)$ of $\alpha$ with each cell $c$ containing the integer $j-i+a$, where $i$ (resp. $j$ ) is the row index (resp. column index) of $c$. In other words, the cells of $Y_{\alpha}(a)$ contain their content shifted by $a$. Each row of $Y_{\alpha}(a)$ can be regarded in a natural way as a segment, and the collection of rows of $Y_{\alpha}(a)$ thus gives rise to a multi-segment

$$
\mathbf{m}(\alpha, a)=\sum_{i=1}^{r}\left[a-i+1, a-i+\alpha_{i}\right] .
$$

Then $S_{\alpha}\left(t^{a}\right)$ is isomorphic to $L_{\mathbf{m}(\alpha, a)}$ Ch.

Example 6 Among the irreducible modules of Example 4 the only evaluation module is $L_{\mathbf{m}_{4}}$ which is isomorphic to $S_{(2,2)}(t)$. 
2.5 Given a decomposition $m=m_{1}+m_{2}$ of $m$ and some $\widehat{H}_{m_{i}}$-modules $M_{i}(i=1,2)$, one can form the induced module

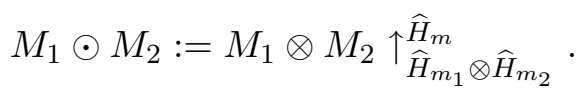

Here $\widehat{H}_{m_{1}} \otimes \widehat{H}_{m_{2}}$ is identified to the subalgebra $\widehat{H}_{\left(m_{1}, m_{2}\right)}$ of $\widehat{H}_{m}$. Suppose that the modules $M_{i}$ are simple, i.e. $M_{i}=L_{\mathbf{m}_{i}}$ for some multi-segments $\mathbf{m}_{i}$. The problem we are interested in is whether one can formulate in terms of $\mathbf{m}_{1}$ and $\mathbf{m}_{2}$ a necessary and sufficient condition of irreducibility for the induced module $M_{1} \odot M_{2}$.

2.6 Our strategy will be to reformulate this problem in the language of canonical bases. Following Zelevinsky [Ze1, let us introduce

$$
R:=\bigoplus_{m \geqslant 0} R_{m},
$$

where we have put for convenience $R_{0}=\mathbf{C}$. By 2.2 . $\left\{\left[M_{\mathbf{m}}\right] \mid \mathbf{m} \in \mathcal{M}\right\}$ and $\left\{\left[L_{\mathbf{m}}\right] \mid \mathbf{m} \in \mathcal{M}\right\}$ are two bases of the vector space $R$.

The induction product endows $R$ with the structure of an associative algebra:

$$
\left[M_{1}\right]\left[M_{2}\right]:=\left[M_{1} \odot M_{2}\right] .
$$

This algebra is commutative because $M_{1} \odot M_{2}$ and $M_{2} \odot M_{1}$ have the same composition factors with the same multiplicities (Ze1], Theorem 1.9). Zelevinsky has shown that $R$ is in fact the polynomial ring in the variables $\left[L_{\mathbf{s}}\right](\mathbf{s} \in \mathcal{S})$ ([Ze1], Corollary 7.5). Using the restrictions from $\widehat{H}_{m}$ to the subalgebras $\widehat{H}_{(k, m-k)} \cong \widehat{H}_{k} \otimes \widehat{H}_{m-k}$, one can define in a standard way a comultiplication $c$ on $R$, and $R$ endowed with these two operations becomes a graded bialgebra ([Ze1], Proposition 1.7), the grading being given by $\operatorname{deg}\left(R_{m}\right)=m$. In particular $c$ is an algebra homomorphism determined by its expression on the generators $\left[L_{\mathbf{s}}\right]$ :

$$
c\left[L_{[i, j]}\right]=1 \otimes\left[L_{[i, j]}\right]+\sum_{k=i}^{j-1}\left[L_{[i, k]}\right] \otimes\left[L_{[k+1, j]}\right]+\left[L_{[i, j]}\right] \otimes 1,
$$

(Ze1], Proposition 3.4). Note that this formula shows that $c$ is not cocommutative.

On the other hand, let $N_{\infty}$ be the group of upper triangular unipotent $(\mathbf{Z} \times \mathbf{Z})$-matrices with finitely many non-zero entries off the main diagonal. We denote by $t_{i j}(i<j)$ the coordinate function $\left(a_{k l}\right) \in N_{\infty} \mapsto a_{i j}$. Let $A$ be the ring of functions on $N_{\infty}$ which are polynomials in the $t_{i j}$ 's. The multiplication of $N_{\infty}$ induces a natural comultiplication $\delta$ on $A$, given on the generators by

$$
\delta t_{i j}=1 \otimes t_{i j}+\sum_{k=i+1}^{j-1} t_{i k} \otimes t_{k j}+t_{i j} \otimes 1,
$$

and this endows $A$ with the structure of a graded bialgebra, where we put $\operatorname{deg} t_{i j}=j-i$. The following simple but crucial observation immediately follows:

Proposition 7 The graded bialgebras $R$ and $A$ are isomorphic via the map

$$
\Psi\left[L_{[i, j]}\right]=t_{i, j+1} \cdot
$$

In this isomorphism, the class $\left[M_{\mathbf{m}}\right]=\prod_{i \leqslant j}\left[L_{[i, j]}\right]^{m_{i j}}$ of a generalized principal series representation is mapped to the monomial $t_{\mathbf{m}}=\prod_{i \leqslant j} t_{i, j+1}^{m_{i j}}$ in the coordinate functions. 
A similar remark was made in Ze1, 7.6.

In the next section, we shall see that $A$ has a natural quantum deformation $A_{v}$, and that it allows us to define a canonical basis of $A$ by specializing at $v=1$ a canonical basis of $A_{v}$.

\section{Quantum algebras and canonical bases}

We review the definitions of the quantum algebras $U_{v}^{+}, A_{v}$ and of their canonical bases. The main reference for this section are Lu1, BZ1. See also [TV].

3.1 Let $\mathfrak{n}_{\infty}$ denote the Lie algebra of strictly upper triangular $(\mathbf{Z} \times \mathbf{Z})$-matrices with finitely many non-zero entries. The standard basis of matrix units in $\mathfrak{n}_{\infty}$ will be denoted by $\left\{e_{i j}, i<j \in \mathbf{Z}\right\}$, and we write $e_{i}:=e_{i, i+1}$. The enveloping algebra $U^{+}=U\left(\mathfrak{n}_{\infty}\right)$ is generated by the $e_{i}, i \in \mathbf{Z}$, subject to Serre's relations:

$$
\begin{array}{ll}
e_{i} e_{j}=e_{j} e_{i}, & |i-j|>1, \\
e_{i}^{2} e_{j}-2 e_{i} e_{j} e_{i}+e_{j} e_{i}^{2}=0, & |i-j|=1 .
\end{array}
$$

Let $U_{v}^{+}$be the associative $\mathbf{Q}(v)$-algebra generated by elements $E_{i}(i \in \mathbf{Z})$ subject to the relations

$$
\begin{array}{ll}
E_{i} E_{j}=E_{j} E_{i}, & |i-j|>1, \\
E_{i}^{2} E_{j}-\left(v+v^{-1}\right) E_{i} E_{j} E_{i}+E_{j} E_{i}^{2}=0, & |i-j|=1 .
\end{array}
$$

This is the quantum enveloping algebra of $\mathfrak{n}_{\infty}$ in the sense of Drinfeld and Jimbo. It can also be seen as the (twisted) Hall-Ringel algebra associated to an orientation $\Gamma$ of the Dynkin diagram of type $A_{\infty}$. We shall always take for $\Gamma$ the standard orientation $k \longrightarrow k-1, k \in \mathbf{Z}$ (cf. 2.3 ).

Let $\mathbf{N}^{(\mathbf{Z})}$ be the semi-group of sequences $\left(d_{j}\right)_{j \in \mathbf{Z}}$ of non-negative integers with finitely many non-zero terms. Denote by $\alpha_{i}$ the sequence whose $i$ th term is equal to 1 and all other terms are zero. We define a bilinear form on $\mathbf{N}^{(\mathbf{Z})}$ by

$$
\left(\alpha_{i}, \alpha_{j}\right)=\left\{\begin{array}{cl}
2 & \text { if } i=j \\
-1 & \text { if }|i-j|=1 \\
0 & \text { otherwise }
\end{array}\right.
$$

$\mathbf{N}^{(\mathbf{Z})}$ identifies to the positive part of the root lattice of the root system $A_{\infty}$, the $\alpha_{i}$ being the simple roots, and the $\alpha_{i j}:=\alpha_{i}+\alpha_{i+1}+\cdots+\alpha_{j},(i \leqslant j)$, the positive roots. The algebra $U_{v}^{+}$is $\mathbf{N}^{(\mathbf{Z})}$-graded via the weight function $\mathrm{wt}\left(E_{i}\right)=\alpha_{i}$. The homogeneous components of $U_{v}^{+}$are finite-dimensional, and their linear bases are naturally labelled by multi-segments. More precisely, the homogeneous component of weight $\alpha$ of $U_{v}^{+}$has dimension equal to the value $p(\alpha)$ of the Kostant partition function (this follows from the Poincaré-Birkhoff-Witt theorem for $U_{v}^{+}$). This value clearly coincides with the number of multi-segments $\mathbf{m}$ with $\operatorname{wt}(\mathbf{m})=\alpha$.

We shall also use the $\mathbf{N}$-grading of $U_{v}^{+}$defined by $\operatorname{deg}\left(E_{i}\right)=1, i \in \mathbf{Z}$.

3.2 Lusztig Lu1 has defined certain bases of $U_{v}^{+}$associated to orientations of the Dynkin diagram, called PBW-bases (they specialize when $v \mapsto 1$ to bases of $U^{+}$of the type provided by the Poincaré-Birkhoff-Witt theorem). We shall introduce the PBW-basis 
corresponding to the quiver $\Gamma$. Let us describe the vector $E(\mathbf{m})$ of this basis labelled by the multi-segment $\mathbf{m}$. When $\mathbf{m}=[i, j]$ is reduced to a single segment, then $E(\mathbf{m})$ is simply an iterated $v$-bracket. Namely

$$
E([i])=E_{i}, \quad E([i, j])=\left[E_{j},\left[E_{j-1},\left[\cdots,\left[E_{i+1}, E_{i}\right]_{v} \cdots\right]_{v}\right]_{v}\right]_{v},
$$

where $[x, y]_{v}:=x y-v^{-(\alpha, \beta)} y x$ for $x, y \in U_{v}^{+}$with $\operatorname{wt}(x)=\alpha$ and $\operatorname{wt}(y)=\beta$. Thus

$$
E([1,2])=E_{2} E_{1}-v E_{1} E_{2}, \quad E([1,3])=E_{3} E_{2} E_{1}-v E_{3} E_{1} E_{2}-v E_{2} E_{1} E_{3}+v^{2} E_{1} E_{2} E_{3} .
$$

Clearly, $E([i, j])$ is a $v$-analogue of the root vector $(-1)^{j-i} e_{i, j+1}$ of $\mathfrak{n}_{\infty}^{+}$, and we have $\operatorname{wt}\left(E([i, j])=\alpha_{i j}\right.$. Next we introduce a total order on the set $\mathcal{S}$ of segments by

$$
[i, j]<[k, l] \Longleftrightarrow\left\{\begin{array}{l}
j<l \\
\text { or } \\
j=l \text { and } i<k .
\end{array}\right.
$$

(This coincides with the total order on positive roots associated with the quiver $\Gamma$.) Then the element of the PBW-basis indexed by $\mathbf{m}=\sum_{i \leqslant j} m_{i j}[i, j]$ is

$$
E(\mathbf{m})=\prod_{[i, j] \in \mathcal{S}} \frac{1}{\left[m_{i j}\right]_{v} !} E([i, j])^{m_{i j}},
$$

where for $a \in \mathbf{Z}$, we set $[a]_{v}=\left(v^{a}-v^{-a}\right) /\left(v-v^{-1}\right)$ and $[a]_{v} !=[a]_{v}[a-1]_{v} \cdots[2]_{v}$. The arrow indicates that the product is taken in the order $<$ on $\mathcal{S}$.

3.3 To define the canonical basis of $U_{v}^{+}$we consider the $\mathbf{Z}[v]$-lattice

$$
\mathcal{L}:=\bigoplus_{\mathbf{m} \in \mathcal{M}} \mathbf{Z}[v] E(\mathbf{m}) \subset U_{v}^{+}
$$

and the involution $x \mapsto \bar{x}$, defined as the unique ring automorphism of $U_{v}^{+}$such that

$$
\bar{v}=v^{-1}, \quad \overline{E_{i}}=E_{i} .
$$

Lusztig has shown [Lu1] that there exists a unique $\mathbf{Q}(v)$-basis $\{G(\mathbf{m}) \mid \mathbf{m} \in \mathcal{M}\}$ of $U_{v}^{+}$ such that

$$
\overline{G(\mathbf{m})}=G(\mathbf{m}), \quad G(\mathbf{m}) \equiv E(\mathbf{m}) \bmod v \mathcal{L} .
$$

This is Lusztig's canonical basis (or Kashiwara's lower global crystal basis).

Example 8 Consider the homogeneous component of $U_{v}^{+}$of degree $\alpha_{0}+2 \alpha_{1}+\alpha_{2}$. It has dimension 5, and its weight vectors are labelled by the multi-segments ( $c f$. Example 4):

$$
\begin{aligned}
& \mathbf{m}_{1}=[0]+2[1]+[2], \mathbf{m}_{2}=[0]+[1]+[1,2], \mathbf{m}_{3}=[0,1]+[1]+[2], \\
& \mathbf{m}_{4}=[0,1]+[1,2], \mathbf{m}_{5}=[1]+[0,2] .
\end{aligned}
$$

The expansion of the $G\left(\mathbf{m}_{i}\right)$ 's on the PBW-basis is

$$
\left\{\begin{array}{l}
G\left(\mathbf{m}_{1}\right)=E\left(\mathbf{m}_{1}\right) \\
G\left(\mathbf{m}_{2}\right)=E\left(\mathbf{m}_{2}\right)+v^{2} E\left(\mathbf{m}_{1}\right) \\
G\left(\mathbf{m}_{3}\right)=E\left(\mathbf{m}_{3}\right)+v^{2} E\left(\mathbf{m}_{1}\right) \\
G\left(\mathbf{m}_{4}\right)=E\left(\mathbf{m}_{4}\right)+v E\left(\mathbf{m}_{3}\right)+v E\left(\mathbf{m}_{2}\right)+\left(v+v^{3}\right) E\left(\mathbf{m}_{1}\right) \\
G\left(\mathbf{m}_{5}\right)=E\left(\mathbf{m}_{5}\right)+v E\left(\mathbf{m}_{4}\right)+v^{2} E\left(\mathbf{m}_{3}\right)+v^{2} E\left(\mathbf{m}_{2}\right)+v^{4} E\left(\mathbf{m}_{1}\right)
\end{array}\right.
$$

It can be calculated by using, for example, the formulas of [LTV] for the products $E_{i} E(\mathbf{m})$. $\diamond$ 
Lusztig has also given a geometrical description of the canonical basis, in terms of the varieties $\bar{O}_{\mathbf{m}}$ introduced in 2.3 .

Theorem 9 ([Lu1]) The expansion of $G(\mathbf{n})$ on the basis $\{E(\mathbf{m})\}$ is given by

$$
G(\mathbf{n})=\sum_{\mathbf{m} \unlhd \mathbf{n}} K_{\mathbf{m n}}(v) E(\mathbf{m}),
$$

where,

$$
K_{\mathbf{m n}}(v)=v^{\operatorname{dim} O_{\mathbf{n}}-\operatorname{dim} O_{\mathbf{m}}} \sum_{i \geqslant 0} v^{-i} \operatorname{dim} \mathcal{H}^{i}\left(\bar{O}_{\mathbf{n}}\right)_{\mathbf{m}}
$$

In particular $E(\mathbf{m})$ occurs with a nonzero coefficient in the expansion of $G(\mathbf{n})$ if and only if $\mathbf{m} \unlhd \mathbf{n}$ for the partial order $\unlhd$ defined in 2.2. Moreover, the coefficient of $E(\mathbf{m})$ in $G(\mathbf{m})$ is 1 .

The fact that precisely the same graded nilpotent orbits occur in Theorem 5 and Theorem 9 is the deep geometrical fact tying together the p-adic groups $G L_{m}$ and the quantum group $U_{v}^{+}$.

3.4 The canonical basis is 'almost orthonormal' with respect to a scalar product introduced by Kashiwara, which on the PBW-basis is given by

$$
(E(\mathbf{m}), E(\mathbf{n}))=\frac{\left(1-v^{2}\right)^{\operatorname{deg}(\mathbf{m})}}{\prod_{i \leqslant j} \varphi_{m_{i j}}\left(v^{2}\right)} \delta_{\mathbf{m}, \mathbf{n}},
$$

where for $k \in \mathbf{N}$ we set $\varphi_{k}(z)=(1-z)\left(1-z^{2}\right) \cdots\left(1-z^{k}\right)$ (see [LTV], 4.3). By almost orthonormal one means that

$$
(G(\mathbf{m}), G(\mathbf{n})) \equiv \delta_{\mathbf{m}, \mathbf{n}} \bmod v \mathbf{A},
$$

where $\mathbf{A}$ is the subring of $\mathbf{Q}(v)$ consisting of functions regular at $v=0$. We will denote by $\left\{E^{*}(\mathbf{m})\right\}$ and $\left\{G^{*}(\mathbf{m})\right\}$ the adjoint bases of $\{E(\mathbf{m})\}$ and $\{G(\mathbf{m})\}$ with respect to this scalar product. Since $\{E(\mathbf{m})\}$ is orthogonal, we see that $\left\{E^{*}(\mathbf{m})\right\}$ is simply a rescaling of $\{E(\mathbf{m})\}$, namely

$$
E^{*}(\mathbf{m})=\frac{\prod_{i \leqslant j} \varphi_{m_{i j}}\left(v^{2}\right)}{\left(1-v^{2}\right)^{\operatorname{deg}(\mathbf{m})}} E(\mathbf{m})=\prod_{[i, j] \in \mathcal{S}} v^{\left(\begin{array}{c}
m_{i j} \\
2
\end{array}\right)} E^{*}([i, j])^{m_{i j}} .
$$

It follows from Theorem 9 that

$$
E^{*}(\mathbf{m})=\sum_{\mathbf{m} \unlhd \mathbf{n}} K_{\mathbf{m n}}(v) G^{*}(\mathbf{n}) .
$$

So if we know the expansion of the canonical basis $\{G(\mathbf{m})\}$ on the PBW-basis, we can obtain the expansion of the dual canonical basis $\left\{G^{*}(\mathbf{m})\right\}$ on the dual PBW-basis by solving this triangular system of linear equations. (For a better algorithm, see below Section 4) In particular, we see that $E^{*}(\mathbf{n})$ occurs in the expansion of $G^{*}(\mathbf{m})$ only if $\mathbf{m} \unlhd \mathbf{n}$, and the coefficient of $E^{*}(\mathbf{m})$ in $G^{*}(\mathbf{m})$ is 1 . Hence for a single segment $\mathbf{s} \in \mathcal{S}$ we have $G^{*}(\mathbf{s})=E^{*}(\mathbf{s})$. 
Example 10 Retaining the notation of Example 8] we have

$$
\left\{\begin{array}{l}
E^{*}\left(\mathbf{m}_{5}\right)=G^{*}\left(\mathbf{m}_{5}\right) \\
E^{*}\left(\mathbf{m}_{4}\right)=G^{*}\left(\mathbf{m}_{4}\right)+v G^{*}\left(\mathbf{m}_{5}\right) \\
E^{*}\left(\mathbf{m}_{3}\right)=G^{*}\left(\mathbf{m}_{3}\right)+v G^{*}\left(\mathbf{m}_{4}\right)+v^{2} G^{*}\left(\mathbf{m}_{5}\right) \\
E^{*}\left(\mathbf{m}_{2}\right)=G^{*}\left(\mathbf{m}_{2}\right)+v G^{*}\left(\mathbf{m}_{4}\right)+v^{2} G^{*}\left(\mathbf{m}_{5}\right) \\
E^{*}\left(\mathbf{m}_{1}\right)=G^{*}\left(\mathbf{m}_{1}\right)+v^{2} G^{*}\left(\mathbf{m}_{2}\right)+v^{2} G^{*}\left(\mathbf{m}_{3}\right)+\left(v+v^{3}\right) G^{*}\left(\mathbf{m}_{4}\right)+v^{4} G^{*}\left(\mathbf{m}_{5}\right)
\end{array}\right.
$$

and

$$
\left\{\begin{array}{l}
G^{*}\left(\mathbf{m}_{5}\right)=E^{*}\left(\mathbf{m}_{5}\right) \\
G^{*}\left(\mathbf{m}_{4}\right)=E^{*}\left(\mathbf{m}_{4}\right)-v E^{*}\left(\mathbf{m}_{5}\right) \\
G^{*}\left(\mathbf{m}_{3}\right)=E^{*}\left(\mathbf{m}_{3}\right)-v E^{*}\left(\mathbf{m}_{4}\right) \\
G^{*}\left(\mathbf{m}_{2}\right)=E^{*}\left(\mathbf{m}_{2}\right)-v E^{*}\left(\mathbf{m}_{4}\right) \\
G^{*}\left(\mathbf{m}_{1}\right)=E^{*}\left(\mathbf{m}_{1}\right)-v^{2} E^{*}\left(\mathbf{m}_{2}\right)-v^{2} E^{*}\left(\mathbf{m}_{3}\right)+\left(v^{3}-v\right) E^{*}\left(\mathbf{m}_{4}\right)+v^{2} E^{*}\left(\mathbf{m}_{5}\right) .
\end{array}\right.
$$

3.5 Let $A_{v}$ be the quantum analogue of the algebra $A$ of polynomials in the coordinate functions $t_{i j}$ of the group $N_{\infty}$. We denote by $T_{i j}(i<j)$ the $v$-analogue of $t_{i j}$ (there should be no risk of confusing the $T_{i j}$ 's with the generators $T_{i}$ of $\widehat{H}_{m}$ ). It is often convenient to write $T_{i j}=0$ for $i>j, T_{i i}=1$, and to index the non-trivial $T_{i j}$ 's by segments, that is, $T_{i j}=T_{[i, j-1]}$, for $i<j$.

The commutation relations satisfied by the $T_{\mathbf{s}},(\mathbf{s} \in \mathcal{S})$ are the following ([BZ1], Proposition 3.11). Let $\mathbf{s}$ and $\mathbf{s}^{\prime}$ be two segments such that $\mathbf{s}^{\prime}>\mathbf{s}$ for the total ordering defined in 3.2, Then

$$
T_{\mathbf{s}^{\prime}} T_{\mathbf{s}}= \begin{cases}v^{-\left(\operatorname{wt}\left(\mathbf{s}^{\prime}\right), \operatorname{wt}(\mathbf{s})\right)}\left(T_{\mathbf{s}} T_{\mathbf{s}^{\prime}}+\left(v^{-1}-v\right) T_{\mathbf{t}^{\prime}} T_{\mathbf{t}}\right) & \text { if } \mathbf{s} \text { and } \mathbf{s}^{\prime} \text { are linked, } \\ v^{-\left(\operatorname{wt}\left(\mathbf{s}^{\prime}\right), \operatorname{wt}(\mathbf{s})\right)} T_{\mathbf{s}} T_{\mathbf{s}^{\prime}} & \text { otherwise. }\end{cases}
$$

As in 2.2 in the case where $\mathbf{s}, \mathbf{s}^{\prime}$ are linked, we have put $\mathbf{t}=\mathbf{s} \cup \mathbf{s}^{\prime}$ and $\mathbf{t}^{\prime}=\mathbf{s} \cap \mathbf{s}^{\prime}$. When $\mathbf{t}^{\prime}=\emptyset$, we understand $T_{\mathbf{t}^{\prime}}=1$.

In fact, as proved in [BZ1, the algebras $U_{v}^{+}$and $A_{v}$ are isomorphic, the isomorphism $\Phi$ being given by $\Phi\left(E_{i}\right)=T_{[i]}=T_{i, i+1}$. More generally, we have $\Phi\left(E^{*}([i, j])\right)=T_{[i, j]}$ (which motivates the notation introduced above), so that

$$
\Phi\left(E^{*}(\mathbf{m})\right)=\prod_{[i, j] \in \mathcal{S}} v^{\left(\begin{array}{c}
m_{i j} \\
2
\end{array}\right)} T_{[i, j]}^{m_{i j}}
$$

For $\mathbf{m} \in \mathcal{M}$, we shall write

$$
T_{\mathbf{m}}=\prod_{[i, j] \in \mathcal{S}} T_{[i, j]}^{m_{i j}}
$$

Relations (2) can be regarded as a set of straightening rules for computing the expansion of an arbitrary polynomial in the $T_{i j}$ 's on the basis $\left\{T_{\mathbf{m}}\right\}$. 
3.6 Although the algebras $U_{v}^{+}$and $A_{v}$ are isomorphic, their natural specializations at $v=1$ are not. Indeed, to specialize $U_{v}^{+}$one first considers the integral form

$$
U_{v, \mathbf{Z}}^{+}=\bigoplus_{\mathbf{m} \in \mathcal{M}} \mathbf{Z}\left[v, v^{-1}\right] E(\mathbf{m})
$$

and then one sets $U_{1}^{+}=\mathbf{C} \otimes_{\mathbf{Z}\left[v, v^{-1}\right]} U_{v, \mathbf{Z}}^{+}$, where $\mathbf{C}$ is regarded as a $\mathbf{Z}\left[v, v^{-1}\right]$-module via $v \mapsto 1$. Clearly, $U_{1}^{+} \simeq U^{+}$via $1 \otimes E_{i} \mapsto e_{i}$. Whereas for $A_{v}$ one takes

$$
A_{v, \mathbf{Z}}=\bigoplus_{\mathbf{m} \in \mathcal{M}} \mathbf{Z}\left[v, v^{-1}\right] T_{\mathbf{m}}
$$

and then $A_{1}=\mathbf{C} \otimes_{\mathbf{Z}\left[v, v^{-1}\right]} A_{v, \mathbf{Z}}$, which is isomorphic to $A$ by $1 \otimes T_{i j} \mapsto t_{i j}$. Thus, $U_{v}^{+}$ specializes to the non-commutative enveloping algebra $U^{+}$, while $A_{v}$ specializes to the commutative algebra of polynomial functions $A$.

Example 11 In $U_{v}^{+}$we have

$$
E([1,2])=E_{2} E_{1}-v E_{1} E_{2}
$$

which gives in $U^{+}$the familiar relation $-e_{13}=e_{23} e_{12}-e_{12} e_{23}$. Relation (3) is transformed under $\Phi$ into

$$
\left(1-v^{2}\right) T_{[1,2]}=T_{[2]} T_{[1]}-v T_{[1]} T_{[2]}
$$

which specializes in $A$ to $t_{23} t_{12}=t_{12} t_{23}$.

In the sequel the algebras $U_{v}^{+}$and $A_{v}$ will in general be identified via $\Phi$, and we shall distinguish between them only when the specialization $v \mapsto 1$ is considered. In particular, the basis $\left\{G^{*}(\mathbf{m})\right\}$ of $U_{v}^{+}$gets identified under $\Phi$ to the dual canonical basis (or string basis) of $A_{v}$ studied by Berenstein and Zelevinsky in BZ1, BZ2.

3.7 The bases $\{E(\mathbf{m})\}$ and $\{G(\mathbf{m})\}$ (resp. $\left\{E^{*}(\mathbf{m})\right\}$ and $\left\{G^{*}(\mathbf{m})\right\}$ ) give rise at $v=1$ to well-defined bases of $U^{+}$(resp. A). We denote them by $\{e(\mathbf{m})\},\{g(\mathbf{m})\},\left\{e^{*}(\mathbf{m})\right\}$ and $\left\{g^{*}(\mathbf{m})\right\}$, respectively. We put $\mathcal{B}_{v}:=\left\{G^{*}(\mathbf{m})\right\}$ and $\mathcal{B}:=\left\{g^{*}(\mathbf{m})\right\}$. It follows from Equation (11) that

$$
e^{*}(\mathbf{m})=\sum_{\mathbf{m} \unlhd \mathbf{n}} K_{\mathbf{m n}} g^{*}(\mathbf{n}) .
$$

Hence, comparing with Theorem [5] we obtain

Theorem 12 Under the isomorphism $\Psi: R \longrightarrow A$ of Proposition 7 , the class of the simple module $L_{\mathbf{m}}$ is mapped to the element $g_{\mathbf{m}}^{*}$ of $\mathcal{B}$.

We note that Ariki has proved a much more general result $\mathbf{A r}$. It describes in a similar way the simple modules of an infinite family of finite-dimensional quotients of $\widehat{H}_{m}$ (the so-called cyclotomic Hecke algebras), and it also handles the case when $t$ is an $\ell$ th root of unity. For an application to the $\ell$-modular representation theory of $G L_{m}$, see [LTV].

Proposition 17 and Theorem 12 imply immediately the following criterion of irreducibility for an induction product of simple modules.

Theorem 13 Let $\mathbf{m}, \mathbf{n}$ be two multi-segments of degree $m$ and $n$ respectively, and $L_{\mathbf{m}}, L_{\mathbf{n}}$ be the corresponding simple modules over $\widehat{H}_{m}$ and $\widehat{H}_{n}$. The induction product $L_{\mathbf{m}} \odot L_{\mathbf{n}}$ is a simple $\widehat{H}_{m+n}$-module if and only if the product $g^{*}(\mathbf{m}) g^{*}(\mathbf{n})$ belongs to $\mathcal{B}$. 
Example 14 Take $\mathbf{m}=[1]+[2,3]$ and $\mathbf{n}=[2]+[3,4]$. Then, using for example the algorithm described in the next section, one calculates

$$
G^{*}(\mathbf{m}) G^{*}(\mathbf{n})=v^{-1} G^{*}\left(\mathbf{p}_{1}\right)+G^{*}\left(\mathbf{p}_{2}\right)+G^{*}\left(\mathbf{p}_{3}\right)+v G^{*}\left(\mathbf{p}_{4}\right)+G^{*}\left(\mathbf{p}_{5}\right),
$$

where $\mathbf{p}_{1}=[1]+[2]+[2,3]+[3,4], \mathbf{p}_{2}=[1]+[2]+[3]+[2,4], \mathbf{p}_{3}=[1,2]+[2,3]+[3,4]$, $\mathbf{p}_{4}=[1,2]+[3]+[2,4], \mathbf{p}_{5}=[1,3]+[2,4]$. Therefore

$$
g^{*}(\mathbf{m}) g^{*}(\mathbf{n})=g^{*}\left(\mathbf{p}_{1}\right)+g^{*}\left(\mathbf{p}_{2}\right)+g^{*}\left(\mathbf{p}_{3}\right)+g^{*}\left(\mathbf{p}_{4}\right)+g^{*}\left(\mathbf{p}_{5}\right),
$$

hence $L_{\mathbf{m}} \odot L_{\mathbf{n}}$ is not irreducible (which agrees with Corollary 39 below) and its composition factors are $L_{\mathbf{p}_{1}}, \ldots, L_{\mathbf{p}_{5}}$, each of them appearing with multiplicity 1 .

Proposition 15 The product $g^{*}(\mathbf{m}) g^{*}(\mathbf{n})$ belongs to $\mathcal{B}$ if and only if there exists $k \in \mathbf{Z}$ such that $v^{k} G^{*}(\mathbf{m}) G^{*}(\mathbf{n})$ belongs to $\mathcal{B}_{v}$.

Proof - Given three multi-segments $\mathbf{m}, \mathbf{n}, \mathbf{p}$, define the coefficient $\alpha_{\mathbf{m} \mathbf{n}}^{\mathbf{p}}(v)$ by

$$
G^{*}(\mathbf{m}) G^{*}(\mathbf{n})=\sum_{\mathbf{p}} \alpha_{\mathbf{m n}}^{\mathbf{p}}(v) G^{*}(\mathbf{p}) .
$$

Using his geometrical description of the canonical basis, Lusztig ( $\mathbf{L u 2}$, 11.5) has proved the positivity of the $\alpha_{\mathbf{m n}}^{\mathbf{p}}(v)$, namely

$$
\alpha_{\mathbf{m n}}^{\mathbf{p}}(v) \in \mathbf{N}\left[v, v^{-1}\right] .
$$

Now

$$
g^{*}(\mathbf{m}) g^{*}(\mathbf{n})=\sum_{\mathbf{p}} \alpha_{\mathbf{m} \mathbf{n}}^{\mathbf{p}}(1) g^{*}(\mathbf{p}),
$$

so if $g^{*}(\mathbf{m}) g^{*}(\mathbf{n})=g^{*}(\mathbf{q})$, then $\alpha_{\mathbf{m n}}^{\mathbf{p}}(1)=\delta_{\mathbf{p q}}$ and all $\alpha_{\mathbf{m} \mathbf{n}}^{\mathbf{p}}(v)$ are zero except $\alpha_{\mathbf{m} \mathbf{n}}^{\mathbf{q}}(v)$ which can only be a power of $v$. The converse is obvious.

\section{Calculation of the dual canonical basis}

4.1 The basis $\left\{G^{*}(\mathbf{m})\right\}$ can be characterized by two conditions similar to those defining $\{G(\mathbf{m})\}$. Let

$$
\mathcal{L}^{*}:=\bigoplus_{\mathbf{m} \in \mathcal{M}} \mathbf{Z}[v] E^{*}(\mathbf{m}),
$$

and let $\tau$ denote the anti-automorphism of $U_{v}^{+}$such that $\tau\left(E_{i}\right)=E_{i}$.

Proposition 16 Let $\mathbf{m} \in \mathcal{M}$ and write $|\mathbf{m}|^{2}:=(\operatorname{wt}(\mathbf{m}), \operatorname{wt}(\mathbf{m}))$. Then $G^{*}(\mathbf{m})$ is the unique homogeneous element of degree $\mathrm{wt}(\mathbf{m})$ of $U_{v}^{+}$satisfying

$$
\overline{G^{*}(\mathbf{m})}=v^{\operatorname{deg}(\mathbf{m})-\frac{1}{2}|\mathbf{m}|^{2}} \tau\left(G^{*}(\mathbf{m})\right), \quad G^{*}(\mathbf{m}) \equiv E^{*}(\mathbf{m}) \bmod v \mathcal{L}^{*} .
$$


Proof - That $G^{*}(\mathbf{m})$ satisfies the second condition is obvious from the definitions. That it also satisfies the first condition was shown by Reineke [Re], Lemma 4.3. (Our power of $v$ is different because we use a different normalization of the scalar product.) Let us prove unicity. Suppose that $x \in U_{v}^{+}$has weight $\mathrm{wt}(\mathbf{m})$ and satisfies $x \in E^{*}(\mathbf{m})+v \mathcal{L}^{*}$. Then

$$
x=G^{*}(\mathbf{m})+\sum_{\mathrm{wt}(\mathbf{n})=\mathrm{wt}(\mathbf{m}),} \alpha_{\mathbf{n}}(v) G^{*}(\mathbf{n})
$$

for some $\alpha_{\mathbf{n}}(v) \in v \mathbf{Z}[v]$. If moreover $\bar{x}=v^{\operatorname{deg}(\mathbf{m})-\frac{1}{2}|\mathbf{m}|^{2}} \tau(x)$ then $\alpha_{\mathbf{n}}\left(v^{-1}\right)=\alpha_{\mathbf{n}}(v)$, which forces $\alpha_{\mathbf{n}}(v)=0$.

The following relation satisfied by the coefficients $\alpha_{\mathbf{m n}}^{\mathbf{p}}(v)$ defined in (15) will be important in the sequel.

Corollary 17 ([Re $]$ ) For $\mathbf{m}, \mathbf{n}, \mathbf{p} \in \mathcal{M}$,

$$
\alpha_{\mathbf{n m}}^{\mathbf{p}}(v)=v^{-(\operatorname{wt}(\mathbf{m}), \operatorname{wt}(\mathbf{n}))} \alpha_{\mathbf{m n}}^{\mathbf{p}}\left(v^{-1}\right) .
$$

Proof - On the one hand,

$$
\begin{aligned}
\overline{G^{*}(\mathbf{m}) G^{*}(\mathbf{n})} & =v^{\operatorname{deg}(\mathbf{m})+\operatorname{deg}(\mathbf{n})-1 / 2\left(|\mathbf{m}|^{2}+|\mathbf{n}|^{2}\right)} \tau\left(G^{*}(\mathbf{m})\right) \tau\left(G^{*}(\mathbf{n})\right) \\
& =v^{\operatorname{deg}(\mathbf{m})+\operatorname{deg}(\mathbf{n})-1 / 2\left(|\mathbf{m}|^{2}+|\mathbf{n}|^{2}\right)} \tau\left(G^{*}(\mathbf{n}) G^{*}(\mathbf{m})\right) \\
& =v^{\operatorname{deg}(\mathbf{m})+\operatorname{deg}(\mathbf{n})-1 / 2\left(|\mathbf{m}|^{2}+|\mathbf{n}|^{2}\right)} \sum_{\mathbf{p}} \alpha_{\mathbf{n} \mathbf{m}}^{\mathbf{p}}(v) \tau\left(G^{*}(\mathbf{p})\right)
\end{aligned}
$$

On the other hand,

$$
\begin{aligned}
\overline{G^{*}(\mathbf{m}) G^{*}(\mathbf{n})} & =\sum_{\mathbf{p}} \alpha_{\mathbf{m} \mathbf{n}}^{\mathbf{p}}\left(v^{-1}\right) \overline{G^{*}(\mathbf{p})} \\
& =\sum_{\mathbf{p}} \alpha_{\mathbf{m} \mathbf{n}}^{\mathbf{p}}\left(v^{-1}\right) v^{\operatorname{deg}(\mathbf{p})-1 / 2|\mathbf{p}|^{2}} \tau\left(G^{*}(\mathbf{p})\right),
\end{aligned}
$$

and the result follows by comparison, since $\operatorname{wt}(\mathbf{p})=\operatorname{wt}(\mathbf{m})+\operatorname{wt}(\mathbf{n})$ for any non-zero coefficient $\alpha_{\mathbf{m} n}^{\mathbf{p}}(v)$.

4.2 Let $\mathfrak{R}$ be the sub-ring of $\mathbf{Z}\left[v, v^{-1}\right]$ consisting of the Laurent polynomials invariant under $v \mapsto v^{-1}$. We set $\mathfrak{V}:=\bigoplus_{\mathbf{m} \in \mathcal{M}} \mathfrak{R} G^{*}(\mathbf{m})$. Then, by Proposition [16, $\mathfrak{V}$ may be described as the $\mathfrak{R}$-lattice whose homogeneous elements $x$ satisfy

$$
\bar{x}=v^{\operatorname{deg}(x)-\frac{1}{2}|\operatorname{wt}(x)|^{2}} \tau(x),
$$

and the vector $G^{*}(\mathbf{m})$ is characterized by

$$
G^{*}(\mathbf{m}) \in \mathfrak{V}, \quad G^{*}(\mathbf{m}) \equiv E^{*}(\mathbf{m}) \bmod v \mathcal{L}^{*} .
$$

Let us describe an algorithm similar to that of [LLT] for computing the transition matrix from $\left\{E^{*}(\mathbf{m})\right\}$ to $\left\{G^{*}(\mathbf{m})\right\}$. Given two multi-segments $\mathbf{m}=\sum_{\mathbf{s} \in \mathcal{S}} m_{\mathbf{s}} \mathbf{s}$ and $\mathbf{n}=$ $\sum_{\mathbf{s} \in \mathcal{S}} n_{\mathbf{s}} \mathbf{s}$, define

$$
\begin{aligned}
b(\mathbf{m}, \mathbf{n}) & :=\sum_{\mathbf{s}^{\prime}>\mathbf{s}} m_{\mathbf{s}^{\prime}} n_{\mathbf{s}}\left(\operatorname{wt}(\mathbf{s}), \operatorname{wt}\left(\mathbf{s}^{\prime}\right)\right)+\sum_{\mathbf{s}} m_{\mathbf{s}} n_{\mathbf{s}} \\
& =\sum_{\mathbf{s}^{\prime}>\mathbf{s}} m_{\mathbf{s}^{\prime}} n_{\mathbf{s}}\left(\operatorname{wt}(\mathbf{s}), \operatorname{wt}\left(\mathbf{s}^{\prime}\right)\right)+\frac{1}{2} \sum_{\mathbf{s}} m_{\mathbf{s}} n_{\mathbf{s}}(\operatorname{wt}(\mathbf{s}), \operatorname{wt}(\mathbf{s})) .
\end{aligned}
$$


Clearly we have

$$
b(\mathbf{m}, \mathbf{n})+b(\mathbf{n}, \mathbf{m})=(\operatorname{wt}(\mathbf{m}), \operatorname{wt}(\mathbf{n})) .
$$

It follows from the straightening relations (2) satisfied by the elements $T_{\mathbf{s}}, \mathbf{s} \in \mathcal{S}$ that

$$
E^{*}(\mathbf{m}) E^{*}(\mathbf{n})=v^{-b(\mathbf{m}, \mathbf{n})} E^{*}(\mathbf{m}+\mathbf{n})+\text { higher terms },
$$

where by 'higher terms' we mean a linear combination of $E^{*}(\mathbf{p})$ with $\mathbf{m}+\mathbf{n} \triangleleft \mathbf{p}$. We know that $E^{*}\left(\mathbf{m}^{\prime}\right)$ occurs in the expansion of $G^{*}(\mathbf{m})$ only if $\mathbf{m} \unlhd \mathbf{m}^{\prime}$. It follows that $\alpha_{\mathbf{m} \mathbf{n}}^{\mathbf{p}}(v) \neq 0$ only if $\mathbf{m}+\mathbf{n} \unlhd \mathbf{p}$, and for $\mathbf{p}=\mathbf{m}+\mathbf{n}$,

$$
\alpha_{\mathbf{m n}}^{\mathbf{m}+\mathbf{n}}=v^{-b(\mathbf{m}, \mathbf{n})} .
$$

Reineke $[\mathbf{R e}$ has given an interesting representation-theoretical interpretation of $b(\mathbf{m}, \mathbf{n})$, namely, if $M$ and $N$ denote respectively the representations of the quiver $\Gamma$ of isomorphism type $\mathbf{m}$ and $\mathbf{n}$, then

$$
b(\mathbf{m}, \mathbf{n})=\operatorname{dim} \operatorname{Hom}(N, M)-\operatorname{dim} \operatorname{Ext}(M, N) .
$$

Now, introduce the following element of $U_{v}^{+}$:

$$
U(\mathbf{m}, \mathbf{n}):=\frac{1}{v-v^{-1}}\left(v^{b(\mathbf{m}, \mathbf{n})+1} G^{*}(\mathbf{m}) G^{*}(\mathbf{n})-v^{b(\mathbf{n}, \mathbf{m})-1} G^{*}(\mathbf{n}) G^{*}(\mathbf{m})\right) .
$$

Proposition 18 Writing $U(\mathbf{m}, \mathbf{n})=\sum_{\mathbf{p}} \beta_{\mathbf{m}, \mathbf{n}}^{\mathbf{p}}(v) G^{*}(\mathbf{p})$, we have

(i) $\quad \beta_{\mathbf{m} \mathbf{n}}^{\mathbf{p}}(v) \in \mathbf{Z}\left[v, v^{-1}\right]$ and $\beta_{\mathbf{m n}}^{\mathbf{m}+\mathbf{n}}(v)=1$,

(ii) $\beta_{\mathbf{m} \mathbf{n}}^{\mathbf{p}}(v) \neq 0$ only if $\mathbf{m}+\mathbf{n} \unlhd \mathbf{p}$,

(iii) $\beta_{\mathbf{m n}}^{\mathbf{p}}\left(v^{-1}\right)=\beta_{\mathbf{m n}}^{\mathbf{p}}(v)$.

Proof - Everything follows immediately from Corollary 17 and relations (8) and (9).

Note that if

$$
G^{*}(\mathbf{m}) G^{*}(\mathbf{n})=v^{c(\mathbf{m}, \mathbf{n})} G^{*}(\mathbf{n}) G^{*}(\mathbf{m}),
$$

then it follows from (9) that $c(\mathbf{m}, \mathbf{n})=b(\mathbf{m}, \mathbf{n})-b(\mathbf{m}, \mathbf{n})$. Hence in such a case $U(\mathbf{m}, \mathbf{n})$ reduces to

$$
U(\mathbf{m}, \mathbf{n})=v^{b(\mathbf{m}, \mathbf{n})} G^{*}(\mathbf{m}) G^{*}(\mathbf{n}) .
$$

We can now easily compute the expansion of the dual canonical basis $\left\{G^{*}(\mathbf{n})\right\}$ on the dual PBW-basis by induction on $\operatorname{deg}(\mathbf{n})$. Suppose that $\left\{G^{*}(\mathbf{n})\right\}$ has already been calculated for $\operatorname{deg}(\mathbf{n}) \leqslant n$. Let $\mathbf{m}$ be a multi-segment of degree $n+1$. If $\mathbf{m}$ consists of a single segment, we know that $G^{*}(\mathbf{m})=E^{*}(\mathbf{m})$. If not, write $\mathbf{m}=\mathbf{m}_{1}+\mathbf{m}_{2}$ for some non-empty multi-segments $\mathbf{m}_{1}$ and $\mathbf{m}_{2}$. (For example, one can take for $\mathbf{m}_{2}$ the largest segment occuring in $\mathbf{m}$ with a non-zero multiplicity). Then the expansions of $G^{*}\left(\mathbf{m}_{1}\right)$ and $G^{*}\left(\mathbf{m}_{2}\right)$ on the dual PBW-basis are known by induction, and one can calculate the expansion of $V(\mathbf{m}):=U\left(\mathbf{m}_{1}, \mathbf{m}_{2}\right)$ on $\left\{E^{*}(\mathbf{n})\right\}$ by means of the straightening rules of $\oint 3.5$, If $\mathbf{m}$ is a single segment, we set $V(\mathbf{m})=E^{*}(\mathbf{m})$.

Then, by Proposition 18 (i) (ii), the transition matrices from $\left\{G^{*}(\mathbf{m}) \mid \operatorname{deg}(\mathbf{m})=n+1\right\}$ to $\{V(\mathbf{m}) \mid \operatorname{deg}(\mathbf{m})=n+1\}$ and from $\left\{E^{*}(\mathbf{m}) \mid \operatorname{deg}(\mathbf{m})=n+1\right\}$ to $\{V(\mathbf{m}) \mid \operatorname{deg}(\mathbf{m})=$ $n+1\}$ will be both unitriangular if their rows and columns are arranged in any total order 
$\leqslant$ extending the partial order $\unlhd$, and their entries will belong to $\mathbf{Z}\left[v, v^{-1}\right]$. Moreover, by Proposition [18 (iii), $V(\mathbf{m}) \in \mathfrak{V}$. This implies that $\{V(\mathbf{m}) \mid \operatorname{deg}(\mathbf{m})=n+1\}$ is an $\mathfrak{R}$-basis of the degree $n+1$ homogeneous component of $\mathfrak{V}$.

Let $\left\{\mathbf{m}_{1}, \ldots, \mathbf{m}_{r}\right\}$ be the list of all multi-segments of degree $n+1$ and of a given weight $\lambda \in \mathbf{N}^{(\mathbf{Z})}$, arranged in increasing order with respect to $\leqslant$. By Proposition 18 (i) and (ii), $V\left(\mathbf{m}_{r}\right)=G^{*}\left(\mathbf{m}_{r}\right)$. Assume by descending induction on $k$ that the expansions of $G^{*}\left(\mathbf{m}_{k+1}\right), \ldots, G^{*}\left(\mathbf{m}_{r}\right)$ on $\left\{E^{*}(\mathbf{n})\right\}$ are known. Then

$$
G^{*}\left(\mathbf{m}_{k}\right)=V\left(\mathbf{m}_{k}\right)-\sum_{i=k+1}^{r} \gamma_{i}(v) G^{*}\left(\mathbf{m}_{i}\right)
$$

where the coefficients $\gamma_{i}(v)$ are completely determined by the conditions

$$
\gamma_{i}\left(v^{-1}\right)=\gamma_{i}(v), \quad G^{*}\left(\mathbf{m}_{k}\right) \equiv E^{*}\left(\mathbf{m}_{k}\right) \bmod v \mathcal{L}^{*} .
$$

Hence we can obtain the basis $\left\{G^{*}(\mathbf{m})\right\}$ from the previously known basis $\{V(\mathbf{m})\}$ by a simple algorithm.

Example 19 Let us calculate with this algorithm the vectors $G^{*}\left(\mathbf{m}_{i}\right)$ of Example 10. For $i=1, \ldots, 5$ we write $\mathbf{m}_{i}=\mathbf{n}_{i}+\mathbf{s}_{i}$ where $\mathbf{s}_{i}$ is the largest segment of $\mathbf{m}_{i}$. Then, by induction we know that

$$
\left\{\begin{array}{l}
G^{*}\left(\mathbf{n}_{5}\right)=G^{*}([1])=E^{*}([1]) \\
G^{*}\left(\mathbf{n}_{4}\right)=G^{*}([0,1])=E^{*}([0,1]) \\
G^{*}\left(\mathbf{n}_{3}\right)=G^{*}([0,1]+[1])=E^{*}([0,1]+[1]) \\
G^{*}\left(\mathbf{n}_{2}\right)=G^{*}([0]+[1])=E^{*}([0]+[1])-v E^{*}([0,1]) \\
G^{*}\left(\mathbf{n}_{1}\right)=G^{*}([0]+2[1])=E^{*}([0]+2[1])-v^{2} E^{*}([0,1]+[1]) .
\end{array}\right.
$$

On the other hand, since $\mathbf{s}_{i}$ is a segment, $G^{*}\left(\mathbf{s}_{i}\right)=E^{*}\left(\mathbf{s}_{i}\right)$. The vectors $V\left(\mathbf{m}_{i}\right)=U\left(\mathbf{n}_{i}, \mathbf{s}_{i}\right)$ are then calculated and found to be equal to

$$
\left\{\begin{array}{l}
V\left(\mathbf{m}_{5}\right)=E^{*}\left(\mathbf{m}_{5}\right) \\
V\left(\mathbf{m}_{4}\right)=E^{*}\left(\mathbf{m}_{4}\right)+v^{-1} E^{*}\left(\mathbf{m}_{5}\right) \\
V\left(\mathbf{m}_{3}\right)=E^{*}\left(\mathbf{m}_{3}\right)+v^{-1} E^{*}\left(\mathbf{m}_{4}\right)+v^{-2} E^{*}\left(\mathbf{m}_{5}\right) \\
V\left(\mathbf{m}_{2}\right)=E^{*}\left(\mathbf{m}_{2}\right)-v E^{*}\left(\mathbf{m}_{4}\right) \\
V\left(\mathbf{m}_{1}\right)=E^{*}\left(\mathbf{m}_{1}\right)+\left(1+v^{-2}\right) E^{*}\left(\mathbf{m}_{2}\right)-v^{2} E^{*}\left(\mathbf{m}_{3}\right)-v E^{*}\left(\mathbf{m}_{4}\right)-E^{*}\left(\mathbf{m}_{5}\right) .
\end{array}\right.
$$

We see that $V\left(\mathbf{m}_{2}\right)$ and $V\left(\mathbf{m}_{5}\right)$ belong to $\mathcal{B}_{v}$, but the other $V\left(\mathbf{m}_{i}\right)$ 's have to be 'corrected' since they do not specialize to $E^{*}\left(\mathbf{m}_{i}\right)$ when $v \mapsto 0$. This gives

$$
\left\{\begin{aligned}
G^{*}\left(\mathbf{m}_{5}\right) & =V\left(\mathbf{m}_{5}\right)=E^{*}\left(\mathbf{m}_{5}\right) \\
G^{*}\left(\mathbf{m}_{4}\right) & =V\left(\mathbf{m}_{4}\right)-\left(v+v^{-1}\right) G^{*}\left(\mathbf{m}_{5}\right)=E^{*}\left(\mathbf{m}_{4}\right)-v E^{*}\left(\mathbf{m}_{5}\right) \\
G^{*}\left(\mathbf{m}_{3}\right) & =V\left(\mathbf{m}_{3}\right)-\left(v+v^{-1}\right) G^{*}\left(\mathbf{m}_{4}\right)-\left(v^{2}+1+v^{-2}\right) G^{*}\left(\mathbf{m}_{5}\right) \\
& =E^{*}\left(\mathbf{m}_{3}\right)-v E^{*}\left(\mathbf{m}_{4}\right) \\
G^{*}\left(\mathbf{m}_{2}\right) & =V\left(\mathbf{m}_{2}\right)=E^{*}\left(\mathbf{m}_{2}\right)-v E^{*}\left(\mathbf{m}_{4}\right) \\
G^{*}\left(\mathbf{m}_{1}\right) & =V\left(\mathbf{m}_{1}\right)-\left(v^{2}+1+v^{-2}\right) G^{*}\left(\mathbf{m}_{2}\right)-\left(v+v^{-1}\right) G^{*}\left(\mathbf{m}_{4}\right) \\
& =E^{*}\left(\mathbf{m}_{1}\right)-v^{2} E^{*}\left(\mathbf{m}_{2}\right)-v^{2} E^{*}\left(\mathbf{m}_{3}\right)+\left(v^{3}-v\right) E^{*}\left(\mathbf{m}_{4}\right)+v^{2} E^{*}\left(\mathbf{m}_{5}\right) .
\end{aligned}\right.
$$


Note that there are many possible choices for $\{V(\mathbf{m})\}$, some of them giving better approximations of $\left\{G^{*}(\mathbf{m})\right\}$ and therefore more efficient algorithms. For example

$$
V^{\prime}\left(\mathbf{m}_{3}\right)=U([0,1],[1]+[2])=E^{*}\left(\mathbf{m}_{3}\right)-v E^{*}\left(\mathbf{m}_{4}\right)=G^{*}\left(\mathbf{m}_{3}\right)
$$

would be a better choice than $V\left(\mathbf{m}_{3}\right)$. We shall not discuss this question here.

\section{The Berenstein-Zelevinsky conjecture}

5.1 The basis $\mathcal{B}_{v}$ seems to enjoy remarkable multiplicative properties. Let us say that $x$ and $y$ quasi-commute if $x y=v^{n} y x$ for some $n \in \mathbf{Z}$. The following deep conjecture was formulated by Berenstein and Zelevinsky, together with some supporting evidence.

Conjecture 20 ([BZ1] $)$ Let $\mathbf{m}, \mathbf{n} \in \mathcal{M}$. The product $G^{*}(\mathbf{m}) G^{*}(\mathbf{n})$ belongs to $\mathcal{B}_{v}$ up to some power of $v$ if and only if $G^{*}(\mathbf{m})$ and $G^{*}(\mathbf{n})$ quasi-commute.

Clearly, Conjecture 20 would imply that more generally the product $G^{*}\left(\mathbf{m}_{1}\right) \cdots G^{*}\left(\mathbf{m}_{r}\right)$ belongs to $\mathcal{B}_{v}$ up to a power of $v$ if and only if $G^{*}\left(\mathbf{m}_{k}\right)$ and $G^{*}\left(\mathbf{m}_{l}\right)$ quasi-commute for all $1 \leqslant k<l \leqslant r$.

Example 21 For a segment $\mathbf{s}$ we have $G^{*}(\mathbf{s})=E^{*}(\mathbf{s})$. Hence, by Equation (11), we deduce that if $\mathbf{m}=\mathbf{s}_{1}+\cdots+\mathbf{s}_{r}$, the product $G^{*}\left(\mathbf{s}_{1}\right) \cdots G^{*}\left(\mathbf{s}_{r}\right)$ belongs to $\mathcal{B}_{v}$ up to some power of $v$ if and only if $\mathbf{m}$ is maximal for the partial order $\unlhd$, that is, if and only if the $\mathbf{s}_{i}$ are pairwise not linked. On the other hand, by Equation (2), this is equivalent to the fact that the $G^{*}\left(\mathbf{s}_{i}\right)$ pairwise $v$-commute.

One implication of Conjecture 20 readily follows from Corollary 17 [Re. Indeed if $G^{*}(\mathbf{m}) G^{*}(\mathbf{n})=v^{k} G^{*}(\mathbf{p})$ for some $k \in \mathbf{Z}$ and some $\mathbf{p} \in \mathcal{M}$, then $G^{*}(\mathbf{n}) G^{*}(\mathbf{m})=$ $v^{-k-(\operatorname{wt}(\mathbf{m}), \operatorname{wt}(\mathbf{n}))} G^{*}(\mathbf{p})$, which means that $G^{*}(\mathbf{m})$ and $G^{*}(\mathbf{n})$ quasi-commute. Another way of seeing this is by specializing $v$ to 1 . For if $v^{k} G^{*}(\mathbf{p})=G^{*}(\mathbf{m}) G^{*}(\mathbf{n})$, then $g^{*}(\mathbf{p})=$ $g^{*}(\mathbf{m}) g^{*}(\mathbf{n})=g^{*}(\mathbf{n}) g^{*}(\mathbf{m})$, and because of the positivity (6) we have $G^{*}(\mathbf{n}) G^{*}(\mathbf{m})=$ $v^{l} G^{*}(\mathbf{p})$ for some $l \in \mathbf{Z}$.

Recall also from 4.2 that if $G^{*}(\mathbf{m})$ and $G^{*}(\mathbf{n})$ quasi-commute, then

$$
v^{b(\mathbf{m}, \mathbf{n})} G^{*}(\mathbf{m}) G^{*}(\mathbf{n})=U(\mathbf{m}, \mathbf{n}),
$$

and thus $v^{b(\mathbf{m}, \mathbf{n})} G^{*}(\mathbf{m}) G^{*}(\mathbf{n}) \in \mathfrak{V}$.

In view of these remarks, Conjecture 20 may be reduced to the following

Conjecture 22 Let $\mathbf{m}, \mathbf{n} \in \mathcal{M}$. If $G^{*}(\mathbf{m})$ and $G^{*}(\mathbf{n})$ quasi-commute, then

$$
v^{b(\mathbf{m}, \mathbf{n})} G^{*}(\mathbf{m}) G^{*}(\mathbf{n}) \equiv E^{*}(\mathbf{m}+\mathbf{n}) \bmod v \mathcal{L}^{*} .
$$

Example 23 (Compare with Lemma II.8 of [MW].) Let $b<b^{\prime \prime}<e^{\prime}<e<e^{\prime \prime}$ be integers and consider the segments

$$
\mathbf{s}=[b, e], \quad \mathbf{s}^{\prime}=\left[b, e^{\prime}\right], \quad \mathbf{s}^{\prime \prime}=\left[b^{\prime \prime}, e^{\prime \prime}\right] .
$$

We have $\mathbf{s}^{\prime}<\mathbf{s}<\mathbf{s}^{\prime \prime}$, and

$$
G^{*}\left(\mathbf{s}^{\prime}\right)=E^{*}\left(\mathbf{s}^{\prime}\right), \quad G^{*}\left(\mathbf{s}+\mathbf{s}^{\prime \prime}\right)=E^{*}\left(\mathbf{s}+\mathbf{s}^{\prime \prime}\right)-v E^{*}\left(\mathbf{t}+\mathbf{t}^{\prime}\right),
$$


where $\mathbf{t}=\mathbf{s} \cup \mathbf{s}^{\prime \prime}=\left[b, e^{\prime \prime}\right]$ and $\mathbf{t}^{\prime}=\mathbf{s} \cap \mathbf{s}^{\prime \prime}=\left[b^{\prime \prime}, e\right]$. From this, we readily obtain that

$$
G^{*}\left(\mathbf{s}^{\prime}\right) G^{*}\left(\mathbf{s}+\mathbf{s}^{\prime \prime}\right)=E^{*}\left(\mathbf{s}+\mathbf{s}^{\prime}+\mathbf{s}^{\prime \prime}\right)-v E^{*}\left(\mathbf{s}^{\prime}+\mathbf{t}+\mathbf{t}^{\prime}\right)=v G^{*}\left(\mathbf{s}+\mathbf{s}^{\prime \prime}\right) G^{*}\left(\mathbf{s}^{\prime}\right) .
$$

Thus, in this case Conjecture 22 is verified and

$$
G^{*}\left(\mathbf{s}^{\prime}\right) G^{*}\left(\mathbf{s}+\mathbf{s}^{\prime \prime}\right)=G\left(\mathbf{s}^{\prime}+\mathbf{s}+\mathbf{s}^{\prime \prime}\right) .
$$

Hence $L_{\mathbf{s}^{\prime}} \odot L_{\mathbf{s}+\mathbf{s}^{\prime \prime}}$ is irreducible and isomorphic to $L_{\mathbf{s}^{\prime}+\mathbf{s}+\mathbf{s}^{\prime \prime}}$.

5.2 For $k \in \mathbf{Z}$ and $r \in \mathbf{N}^{*}$, let $Q_{k, r}$ denote the sub-semigroup of $\mathbf{N}^{(\mathbf{Z})}$ generated by the simple roots $\alpha_{k}, \alpha_{k+1}, \ldots, \alpha_{k+r-1}$. If $\operatorname{wt}(\mathbf{m})$ and $\operatorname{wt}(\mathbf{n})$ belong to $Q_{k, r}$ for some $k \in \mathbf{Z}$ and $r \leqslant 3$, then Conjecture 22 is true BZ1. Moreover in this case the set $\left\{G^{*}(\mathbf{m}) \mid \operatorname{wt}(\mathbf{m}) \in Q_{k, r}\right\}$ consists of quasi-commuting products of a small number of certain special elements called quantum minors (4 primitive minors for $r=2$ and 12 for $r=3)$.

Given two subsets $I=\left\{i_{1}<\cdots<i_{k}\right\}, J=\left\{j_{1}<\cdots<j_{k}\right\}$ of $\mathbf{Z}$, the quantum minor $\Delta(I, J)$ is defined by

$$
\Delta(I, J):=\sum_{\sigma \in \mathfrak{S}_{k}}(-v)^{\ell(\sigma)} T_{i_{1} j_{\sigma(1)}} \cdots T_{i_{k} j_{\sigma(k)}}
$$

Note that with our convention for $T_{i j}, \Delta(I, J) \neq 0$ only if $i_{r} \leqslant j_{r}(r=1, \ldots, k)$. Moreover, if $i_{r}=j_{r}$ then $\Delta(I, J)$ factors into

$$
\Delta(I, J)=\Delta\left(I^{\prime}, J^{\prime}\right) \Delta\left(I^{\prime \prime}, J^{\prime \prime}\right)
$$

where $I^{\prime}=\left\{i_{1}, \ldots, i_{r-1}\right\}, I^{\prime \prime}=\left\{i_{r+1}, \ldots, i_{k}\right\}, J^{\prime}=\left\{j_{1}, \ldots, j_{r-1}\right\}$ and $J^{\prime \prime}=\left\{j_{r+1}, \ldots, j_{k}\right\}$. One can thus assume that $i_{r} \leqslant j_{r}(r=1, \ldots, k)$ and attach to $\Delta(I, J)$ the multi-segment

$$
\mathbf{m}(I, J)=\sum_{r=1}^{k}\left[i_{r}, j_{r}-1\right]
$$

in which $\left[i_{r}, j_{r}-1\right]$ is omitted if $i_{r}=j_{r}$.

Proposition 24 ([BZ1 $)$ The non-zero quantum minors form a subset of the dual canonical basis. More precisely, $\Delta(I, J)=G^{*}(\mathbf{m}(I, J))$.

In view of Conjecture 22 it is natural to look for a necessary and sufficient condition for two quantum minors to quasi-commute. This problem was solved in $\mathbf{L Z}$ in the case of quantum flag minors. We call quantum flag minors the elements $\Delta(I, J)$ for which $I$ consists of consecutive integers : $I=\{i+1, i+2, \ldots, i+k\}=[i+1, i+k]$. By (10), for such a minor and for all $s \geqslant 0$ we have $\Delta(I, J)=\Delta([i-s, i+k],[i-s, i] \cup J)$. Thus, letting $s$ tend to $\infty$ we get $\Delta(I, J)=\Delta\left(\mathbf{Z}_{\leqslant i+k}, \mathbf{Z}_{\leqslant i} \cup J\right)$. Thus a quantum flag minor may be labelled by a unique subset of $\mathbf{Z}$ of the form $\mathcal{J}=\mathbf{Z}_{\leqslant i} \cup J$, where $J$ is a finite subset of $\mathbf{Z}_{>i}$. It will be convenient to write $\langle\mathcal{J}\rangle$ in place of $\Delta(I, J)$.

Let $A, B$ be finite disjoint subsets of $\mathbf{Z}$ of cardinality $|A|$ and $|B|$. We write $A \prec B$ if $a<b$ for any $a \in A, b \in B$ (in particular if $A$ or $B$ or both are empty). More generally, we write $A \bowtie B$ if one of the following holds:

either $|A| \leqslant|B|$ and $A=A^{\prime} \cup A^{\prime \prime}$ with $A^{\prime} \prec B \prec A^{\prime \prime}$, or $|B| \leqslant|A|$ and $B=B^{\prime} \cup B^{\prime \prime}$ with $B^{\prime} \prec A \prec B^{\prime \prime}$. 
Let now $\mathcal{I}=\mathbf{Z}_{\leqslant i} \cup I$ and $\mathcal{J}=\mathbf{Z}_{\leqslant j} \cup J$ be two infinite subsets as above. Set $I^{\prime}:=\mathcal{I} \backslash \mathcal{J}$ and $J^{\prime}:=\mathcal{J} \backslash \mathcal{I}$. Then $I^{\prime}$ and $J^{\prime}$ are finite disjoint subsets. We say that $\mathcal{I}$ and $\mathcal{J}$ are separated if $I^{\prime} \bowtie J^{\prime}$, and strongly separated if $I^{\prime} \prec J^{\prime}$ or $J^{\prime} \prec I^{\prime}$. We can now state

Proposition 25 ([LZ $)$ The quantum flag minors $\langle\mathcal{I}\rangle$ and $\langle\mathcal{J}\rangle$ quasi-commute if and only if the subsets $\mathcal{I}$ and $\mathcal{J}$ are separated.

Example 26 Let $a \in \mathbf{Z}, \mathcal{I}=\mathbf{Z}_{\leqslant-1} \cup\{1,5\}$ and $\mathcal{J}(a)=\mathbf{Z}_{\leqslant a} \cup\{a+2, a+4\}$. Then $\mathcal{I}$ and $\mathcal{J}$ are not separated for $a= \pm 2$, are separated for $a=0$, and strongly separated in all other cases. Hence the quantum flag minors $\langle\mathcal{I}\rangle$ and $\langle\mathcal{J}(a)\rangle$ quasi-commute for all $a \neq \pm 2$.

\section{The Berenstein-Zelevinsky conjecture for flag minors}

6.1 Consider a product $\pi=\left\langle\mathcal{I}_{1}\right\rangle \cdots\left\langle\mathcal{I}_{r}\right\rangle$ of quantum flag minors. Recall that the sets $\mathcal{I}_{k}$ are of the form $\mathcal{I}_{k}=\mathbf{Z}_{\leqslant a_{k}} \cup I_{k}$ for some finite set $I_{k}$ contained in $\mathbf{Z}_{\geqslant a_{k}}$. This decomposition is not unique, and one could replace $a_{k}$ by any $b \leqslant a_{k}$ and write $\mathcal{I}_{k}=\mathbf{Z}_{\leqslant b} \cup\left(\left[b+1, a_{k}\right] \cup I_{k}\right)$. Hence, taking $b=\min \left\{a_{1}, \ldots, a_{r}\right\}$, we can assume that all $a_{k}$ 's are equal. Moreover, since the translations of indices $T_{i j} \mapsto T_{i+n, j+n}$ extend to algebra automorphisms of $A_{v}$ for all $n \in \mathbf{Z}$, there is no loss of generality in assuming that $a_{k}=0$ for all $k$. So from now on, we will suppose that $\mathcal{I}_{k}=\mathbf{Z}_{\leqslant 0} \cup I_{k}$ with

$$
I_{k}=\left\{i_{1}^{(k)}<i_{2}^{(k)}<\cdots<i_{n_{k}}^{(k)}\right\} \subset \mathbf{Z}_{>0} .
$$

The corresponding multi-segments will be denoted by

$$
\mathbf{m}_{k}=\mathbf{m}\left(\left[1, n_{k}\right], I_{k}\right)=\sum_{j=1}^{n_{k}}\left[j, i_{j}^{(k)}-1\right] .
$$

To the product $\pi=\left\langle\mathcal{I}_{1}\right\rangle \cdots\left\langle\mathcal{I}_{r}\right\rangle$ we associate the multi-segment $\mathbf{m}_{\pi}=\sum_{k=1}^{r} \mathbf{m}_{k}$ and the integer

$$
b_{\pi}=\sum_{1 \leqslant k<l \leqslant r} b\left(\mathbf{m}_{k}, \mathbf{m}_{l}\right)
$$

where $b(\mathbf{m}, \mathbf{n})$ was defined in 4.2 . We also define the word

$$
w_{\pi}=i_{n_{r}}^{(r)} \cdots i_{2}^{(r)} i_{1}^{(r)} i_{n_{r-1}}^{(r-1)} \cdots i_{2}^{(r-1)} i_{1}^{(r-1)} \cdots i_{n_{1}}^{(1)} \cdots i_{2}^{(1)} i_{1}^{(1)}
$$

obtained by reading the sets $I_{r}, \ldots, I_{1}$ successively in decreasing order. Recall that the Robinson-Schensted correspondence maps a word $w$ to a pair $(P(w), Q(w))$ of Young tableaux of the same shape $\lambda$ (see for example $\mathbf{F u}$ ). We denote by $\mu_{\pi}$ the partition conjugate to the shape of $P\left(w_{\pi}\right)$. Finally, we introduce the multi-segment $\mathbf{n}_{\pi}=\sum_{i<j} n_{i j}[i, j]$ where $n_{i j}$ is the number of letters $j+1$ on the $i$ th row of the Young tableau $P\left(w_{\pi}\right)$.

Proposition 27 Suppose that $\mu_{\pi}$ is equal to the non-increasing reordering of $\left(n_{1}, \ldots, n_{r}\right)$. Then $v^{d_{\pi}} \pi \equiv G^{*}\left(\mathbf{n}_{\pi}\right) \bmod v \mathcal{L}^{*}$, for some integer $d_{\pi}$.

The proof of Proposition 27 will be given in Section 7 
Example 28 Let $r=2$ and $I_{1}=\{2,3,5\}, I_{2}=\{1,4\}$. Then

$$
\left\langle\mathcal{I}_{1}\right\rangle=G^{*}([1]+[2]+[3,4]), \quad\left\langle\mathcal{I}_{2}\right\rangle=G^{*}([2,3]) .
$$

We have $w_{\pi}=41532$ and

$$
P\left(w_{\pi}\right)=\begin{array}{|l|l|}
\hline 4 & \\
\hline 3 & 5 \\
\hline 1 & 2 \\
\hline
\end{array}
$$

(Here and in what follows we choose the French orientation for drawing Young tableaux.) Thus, $\mu_{\pi}=(3,2)=\left(n_{1}, n_{2}\right)$, and $\pi$ satisfies the hypothesis of Proposition 27. We have $\mathbf{n}_{\pi}=[1]+[2]+[3]+[2,4]$, and

$$
v \pi=G^{*}([1]+[2]+[3]+[2,4])+v G^{*}([1]+[2]+[2,3]+[3,4]),
$$

which shows that $v \pi \equiv G^{*}\left(\mathbf{n}_{\pi}\right) \bmod v \mathcal{L}^{*}$.

A word $w_{\pi}$ satisfying the hypothesis of Proposition 27 is a frank word, as defined by Lascoux and Schützenberger [LS]. The combinatorics of frank words has already occured in several interesting problems [LS, [FL. Proposition 27 easily yields the following

Theorem 29 Suppose that the sets $\mathcal{I}_{1}, \ldots, \mathcal{I}_{r}$ are pairwise strongly separated. Then

$$
v^{b_{\pi}} \pi=v^{b_{\pi}}\left\langle\mathcal{I}_{1}\right\rangle \cdots\left\langle\mathcal{I}_{r}\right\rangle=G^{*}\left(\mathbf{m}_{\pi}\right)
$$

belongs to the dual canonical basis.

Proof - If two sets $I$ and $J$ are strongly separated then either $I \backslash J \prec J \backslash I$ or $J \backslash I \prec$ $I \backslash J$. Since the quantum minors $\left\langle\mathcal{I}_{k}\right\rangle$ pairwise quasi-commute, we may assume that their indexing is so chosen that for $k<l$ we have $I_{l} \backslash I_{k} \prec I_{k} \backslash I_{l}$. In this case, one checks easily that the $s$ th row of the tableau $P\left(w_{\pi}\right)$ is exactly the reordering of the letters

$$
i_{s}^{\left(a_{1}\right)}, i_{s}^{\left(a_{2}\right)}, \ldots, i_{s}^{\left(a_{t}\right)},
$$

where $\left\{a_{1}, \ldots, a_{t}\right\}$ is the subset of $[1, r]$ consisting of the integers $k$ for which $n_{k} \geqslant s$. Hence, $\mu_{\pi}$ is the reordering of $\left(n_{1}, \ldots, n_{r}\right)$. It is also clear from this description of $P\left(w_{\pi}\right)$ that $\mathbf{m}_{\pi}=\mathbf{n}_{\pi}$ in this case. By Proposition 27, it follows that

$$
v^{d_{\pi}} \pi \equiv E^{*}\left(\mathbf{m}_{\pi}\right) \bmod v \mathcal{L}^{*}
$$

for some $d_{\pi} \in \mathbf{Z}$. Now it follows from (9) that the expansion of $\pi$ on $\left\{E^{*}(\mathbf{m})\right\}$ contains $E^{*}\left(\mathbf{m}_{\pi}\right)$ with coefficient $v^{-b_{\pi}}$. Hence we also have $d_{\pi}=b_{\pi}$. Therefore for $r=2$ we have verified Conjecture 22, and Theorem 29 is proved in this case. Otherwise, putting $\pi^{\prime}=\left\langle\mathcal{I}_{1}\right\rangle \cdots\left\langle\mathcal{I}_{r-1}\right\rangle$ we may suppose by induction on $r$ that

$$
v^{b_{\pi^{\prime}}} \pi^{\prime}=G^{*}\left(\mathbf{m}_{\pi^{\prime}}\right) .
$$

Then $G^{*}\left(\mathbf{m}_{\pi^{\prime}}\right)$ and $\left\langle\mathcal{I}_{r}\right\rangle=G^{*}\left(\mathbf{m}_{r}\right)$ quasi-commute and satisfy

$$
v^{b\left(\mathbf{m}_{\pi^{\prime}}, \mathbf{m}_{r}\right)} G^{*}\left(\mathbf{m}_{\pi^{\prime}}\right) G^{*}\left(\mathbf{m}_{r}\right) \equiv E^{*}\left(\mathbf{m}_{\pi^{\prime}}+\mathbf{m}_{r}\right) \bmod v \mathcal{L}^{*} .
$$

Hence $G^{*}\left(\mathbf{m}_{\pi^{\prime}}\right)$ and $G^{*}\left(\mathbf{m}_{r}\right)$ verify Conjecture 22, and the result is proved. 
6.2 In the case of a product of $r=2$ quantum flag minors, we can drop the hypothesis of strong separation in Theorem 29] and deduce from Proposition 27] the following

Theorem 30 Let $\pi=\langle\mathcal{I}\rangle\langle\mathcal{J}\rangle$ be a product of two quantum flag minors. Then $\pi$ belongs to the dual canonical basis up to a power of $v$ if and only if $\langle\mathcal{I}\rangle$ and $\langle\mathcal{J}\rangle$ quasi-commute.

The proof of Theorem 30 will be given in Section 7

6.3 Finally, using an argument of [KMT, $\mathbf{M T}$ ] about finite-dimensional representations of $U_{v}\left(\widehat{\mathfrak{s l}}_{N}\right)$ as well as Theorem [30, we can improve on Theorem 29] and obtain the main result.

Theorem 31 Let $\pi=\left\langle\mathcal{I}_{1}\right\rangle \cdots\left\langle\mathcal{I}_{r}\right\rangle$ be a product of any number $r$ of quantum flag minors. Then $\pi$ belongs to the dual canonical basis up to a power of $v$ if and only if the minors $\left\langle\mathcal{I}_{k}\right\rangle$ pairwise quasi-commute.

Note that if $\pi$ belongs to $\mathcal{B}_{v}$ up to a power of $v$, this power has to be $v^{b_{\pi}}$. The proof of Theorem 31 will be given in Section 8 .

\section{Proofs of Proposition 27 and Theorem 30}

7.1 We retain the notation of 6.1 Let $N=\max \left(I_{1} \cup \cdots \cup I_{r}\right)$. The quantum minors $\left\langle\mathcal{I}_{1}\right\rangle, \ldots,\left\langle\mathcal{I}_{r}\right\rangle$ belong to the subalgebra of $U_{v}\left(\mathfrak{n}_{\infty}\right)$ generated by $E_{1}, \ldots, E_{N-1}$, which is isomorphic to $U_{v}^{+}\left(\mathfrak{s} l_{N}\right)$. So Proposition 27] can be regarded as a statement about $U_{v}(\mathfrak{g})$, where $\mathfrak{g}=\mathfrak{s l}_{N}$. We denote by $E_{i}, F_{i}, K_{i}(i=1, \ldots, N-1)$ the standard generators of $U_{v}(\mathfrak{g})$. The subalgebras generated by $E_{i}(i=1, \ldots, N-1)$ (resp. by $E_{i}, K_{i}(i=$ $1, \ldots, N-1)$ ) are denoted by $U_{v}(\mathfrak{n})\left(\right.$ resp. $\left.U_{v}(\mathfrak{b})\right)$. The fundamental weights of $\mathfrak{g}$ are denoted by $\Lambda_{1}, \ldots, \Lambda_{N-1}$.

7.2 We recall some general properties of the canonical bases of $U_{v}(\mathfrak{n})$ and of the finitedimensional irreducible $U_{v}(\mathfrak{g})$-modules. Our aim is to express the multiplication of vectors of the dual canonical basis of $U_{v}(\mathfrak{n})$ in terms of the tensor products of vectors of the dual canonical bases of the irreducible modules. The precise relation will be given by 7.2 .7 (18) below.

7.2.1 Let $\lambda$ be an integral dominant weight of $\mathfrak{g}$ and $V(\lambda)$ the irreducible $U_{v}(\mathfrak{g})$-module with highest weight $\lambda$. Let $\mathcal{M}_{N}$ denote the set of multi-segments supported on $[1, N-1]$. The canonical (or lower global) basis of $U_{v}(\mathfrak{n})$ may be identified with the subset of the canonical basis of $U_{v}\left(\mathfrak{n}_{\infty}\right)$ consisting of the vectors $G(\mathbf{m})$ with $\mathbf{m} \in \mathcal{M}_{N}$. Let $u_{\lambda}$ be a fixed lowest weight vector of $V(\lambda)$ (hence $u_{\lambda}$ has weight $w_{0} \lambda$ where $w_{0}$ is the longest element of the Weyl group of $\mathfrak{g})$. It is known that the image of $\left\{G(\mathbf{m}) \mid \mathbf{m} \in \mathcal{M}_{N}\right\}$ under the map

$$
\pi_{\lambda}: U_{v}(\mathfrak{n}) \longrightarrow V(\lambda), \quad x \longrightarrow x u_{\lambda}
$$

is the union of a basis of $V(\lambda)$ with the set $\{0\}$. This basis is called the canonical (or lower global) basis of $V(\lambda)$ and is naturally labelled by the set Tab $(\lambda)$ of Young tableaux of shape $\lambda$ over $[1, N]$. (Here we identify as usual the dominant integral weight $\lambda$ with a partition of length $\leqslant N-1$.) This basis will be denoted by $\{G(t) \mid t \in \operatorname{Tab}(\lambda)\}$. 
7.2.2 Given a left $U_{v}(\mathfrak{g})$-module $M$, we endow $M^{*}=\operatorname{Hom}(M, \mathbf{Q}(v))$ with a left $U_{v}(\mathfrak{g})$ action by setting

$$
(x \phi)(m)=\phi\left(x^{\natural} m\right) \quad x \in U_{v}(\mathfrak{g}), m \in M, \phi \in M^{*},
$$

where $x \mapsto x^{\natural}$ is the anti-automorphism of $U_{v}(\mathfrak{g})$ defined by

$$
E_{i}^{\natural}=F_{i}, \quad F_{i}^{\natural}=E_{i}, \quad K_{i}^{\natural}=K_{i} .
$$

It is known that $V(\lambda)^{*}$ is isomorphic to $V(\lambda)$ as a $U_{v}(\mathfrak{g})$-module. In other words, there is a non-degenerate scalar product on $V(\lambda)$ satisfying

$$
\left(u_{\lambda}, u_{\lambda}\right)=1, \quad(x u, v)=(u, \sigma(x) v), \quad x \in U_{v}(\mathfrak{g}), u, v \in V(\lambda),
$$

and $V(\lambda)^{*}$ can be identified to $V(\lambda)$ via the map $V(\lambda) \longrightarrow V(\lambda)^{*}, x \longrightarrow(x, \cdot)$.

The basis $\mathcal{B}_{v}(\lambda)=\left\{G^{*}(t) \mid t \in \operatorname{Tab}(\lambda)\right\}$ dual to $\{G(t)\}$ is called the dual canonical (or upper global) basis of $V(\lambda)^{*}$ (or $V(\lambda)$ ).

7.2.3 Similarly, Kashiwara's scalar product on $U_{v}(\mathfrak{n})$ (defined as in 3.4) allows to identify $U_{v}(\mathfrak{n})$ to its graded dual $U_{v}(\mathfrak{n})^{*}$, as vector spaces. By composing these maps we obtain an embedding

$$
\psi_{\lambda}: V(\lambda) \simeq V(\lambda)^{*} \stackrel{\pi_{\lambda}^{*}}{\longrightarrow} U_{v}(\mathfrak{n})^{*} \simeq U_{v}(\mathfrak{n})
$$

satisfying $\psi_{\lambda}\left(u_{\lambda}\right)=1$ and $\psi_{\lambda}\left(\mathcal{B}_{v}(\lambda)\right) \subset \mathcal{B}_{v}$, where, as in $3.4 \mathcal{B}_{v}=\left\{G^{*}(\mathbf{m}) \mid \mathbf{m} \in \mathcal{M}_{N}\right\}$ is the basis dual to the canonical basis $\left\{G(\mathbf{m}) \mid \mathbf{m} \in \mathcal{M}_{N}\right\}$.

More precisely, write $\lambda=\sum_{i=1}^{N-1} a_{i} \Lambda_{i}$ and set $\tilde{\lambda}=\sum_{i=1}^{N-1} a_{i} \Lambda_{N-i}$. If $t \in \operatorname{Tab}(\lambda)$ has columns $C_{1}, \ldots, C_{m}$, where $m=\sum_{i} a_{i}$, we define $\tilde{t} \in \operatorname{Tab}(\hat{\lambda})$ as the Young tableau with columns $D_{1}, \ldots, D_{m}$, where $D_{i}=[1, N] \backslash C_{m+1-i}$. (Here, columns of Young tableaux are identified to subsets of $[1, N]$ in a natural way). Finally, we introduce the multi-segment $\mathbf{m}(t)=\sum_{i<j} m_{i j}(t)[i, j]$, where $m_{i j}(t)$ is the number of letters $j+1$ in the $i$ th row of $\tilde{t}$. Then $\psi_{\lambda}\left(G^{*}(t)\right)=G^{*}(\mathbf{m}(t))$.

7.2.4 Let $\lambda$ and $\mu$ be two dominant weights. The tensor product $V(\lambda) \otimes V(\mu)$ is a $U_{v}(\mathfrak{g})$-module via the comultiplication

$$
\begin{aligned}
\Delta E_{i} & =E_{i} \otimes 1+K_{i}^{-1} \otimes E_{i}, \\
\Delta F_{i} & =F_{i} \otimes K_{i}+1 \otimes F_{i}, \\
\Delta K_{i} & =K_{i} \otimes K_{i},
\end{aligned}
$$

and we have the following commutative diagram

$$
\begin{array}{ccc}
U_{v}(\mathfrak{g}) & \stackrel{\Delta}{\longrightarrow} & U_{v}(\mathfrak{g}) \otimes U_{v}(\mathfrak{g}) \\
p_{\lambda+\mu} \downarrow & & \downarrow p_{\lambda} \otimes p_{\mu} \\
V(\lambda+\mu) & \stackrel{i_{\lambda, \mu}}{\longrightarrow} & V(\lambda) \otimes V(\mu)
\end{array}
$$

where $p_{\lambda}: U_{v}(\mathfrak{g}) \longrightarrow V(\lambda), x \longrightarrow x u_{\lambda}$, and $i_{\lambda, \mu}$ is the homomorphism of $U_{v}(\mathfrak{g})$-modules mapping $u_{\lambda+\mu}$ to $u_{\lambda} \otimes u_{\mu}$. Dualizing, we obtain

$$
\begin{array}{ccc}
U_{v}(\mathfrak{g})^{*} \otimes U_{v}(\mathfrak{g})^{*} & \stackrel{m}{\longrightarrow} & U_{v}(\mathfrak{g})^{*} \\
p_{\lambda}^{*} \otimes p_{\mu}^{*} \uparrow & & \uparrow p_{\lambda+\mu}^{*} \\
V(\lambda)^{*} \otimes V(\mu)^{*} & \stackrel{i_{\lambda, \mu}^{*}}{\longrightarrow} V(\lambda+\mu)^{*}
\end{array}
$$


where $m$ is the multiplication dual to $\Delta$, and $V(\lambda)^{*} \otimes V(\mu)^{*} \simeq(V(\lambda) \otimes V(\mu))^{*}$ is endowed with the action induced by the comultiplication

$$
\begin{aligned}
\Delta^{\prime} E_{i} & =E_{i} \otimes K_{i}+1 \otimes E_{i}, \\
\Delta^{\prime} F_{i} & =F_{i} \otimes 1+K_{i}^{-1} \otimes F_{i}, \\
\Delta^{\prime} K_{i} & =K_{i} \otimes K_{i},
\end{aligned}
$$

(this comes from 7.2.2).

7.2.5 In contrast to the case $v=1, U_{v}(\mathfrak{n})$ is not a Hopf subalgebra of $U_{v}(\mathfrak{g})$ : it is not stable under $\Delta$. Nevertheless, one can define a multiplication on $U_{v}(\mathfrak{n})^{*}$ by embedding it into $U_{v}(\mathfrak{b})^{*}$ via $\phi \longrightarrow \tilde{\phi}$, where for $\phi \in U_{v}(\mathfrak{n})^{*}$,

$$
\tilde{\phi}\left(x K_{i}\right)=\phi(x), \quad x \in U_{v}(\mathfrak{n}), i=1, \ldots, N-1 .
$$

It is easy to see that the image of $U_{v}(\mathfrak{n})^{*}$ is a subalgebra of $U_{v}(\mathfrak{b})^{*}$, hence $U_{v}(\mathfrak{n})^{*}$ inherits a multiplication. It is also known that the vector space isomorphism $U_{v}(\mathfrak{n}) \longrightarrow U_{v}(\mathfrak{n})^{*}$ of 7.2 .3 becomes in this way an algebra isomorphism.

7.2.6 The image of $V(\lambda)^{*}$ under $p_{\lambda}^{*}$ can be regarded as a subset of $U_{v}(b)^{*}$. More precisely, let $\phi \in V(\lambda)^{*}$ and set $\bar{\phi}=\pi_{\lambda}^{*}(\phi) \in U_{v}(\mathfrak{n})^{*}, \tilde{\phi}=p_{\lambda}^{*}(\phi) \in U_{v}(\mathfrak{b})^{*}$. For $x \in U_{v}(\mathfrak{n})$, we have

$$
\tilde{\phi}\left(x K_{i}\right)=\phi\left(x K_{i} u_{\lambda}\right)=v^{\left(\alpha_{i}, w_{0} \lambda\right)} \phi\left(x u_{\lambda}\right)=v^{\left(\alpha_{i}, w_{0} \lambda\right)} \bar{\phi}(x) .
$$

Let $Q^{+}=\bigoplus_{i=1}^{N-1} \mathbf{N} \alpha_{i}$ be the monoid generated by the simple roots of $\mathfrak{g}$. We say that $\bar{\phi}$ (or $\tilde{\phi}$ ) has weight $\alpha \in Q^{+}$if $\bar{\phi}$ vanishes on all homogeneous elements of $U_{v}(\mathbf{n})$ of weight $\neq \alpha$.

Lemma 32 Let $\phi \in V(\lambda)^{*}, \psi \in V(\mu)^{*}$ and suppose that $\bar{\psi}$ has weight $\beta$. We have

$$
(\tilde{\phi} \cdot \tilde{\psi})\left(x K_{i}\right)=v^{\left(\alpha_{i}, w_{0}(\lambda+\mu)\right)-\left(\beta, w_{0} \lambda\right)}(\bar{\phi} \cdot \bar{\psi})(x), \quad x \in U_{v}(\mathfrak{n}), i=1, \ldots N-1,
$$

where $\tilde{\phi} \cdot \tilde{\psi}$ denotes multiplication in $U_{v}(\mathfrak{b})^{*}$ and $\bar{\phi} \cdot \bar{\psi}$ multiplication in $U_{v}(\mathfrak{n})^{*}$.

Proof - Let $x \in U_{v}(\mathfrak{n})$ and put $\Delta x=\sum_{(x)} x_{(1)} \otimes x_{(2)}$, where $x_{(1)} \in U_{v}(\mathfrak{b})$ and $x_{(2)} \in$ $U_{v}(\mathfrak{n})$. In view of (12), one can moreover assume that $x_{(2)}$ is homogeneous of weight $\sum_{i=1}^{N-1} a_{i} \alpha_{i}$, and $x_{(1)}=x_{(1)}^{\prime} \prod_{i=1}^{N-1} K_{i}^{-a_{i}}$ with $x_{(1)}^{\prime} \in U_{v}(\mathfrak{n})$. Then

$$
\begin{aligned}
(\tilde{\phi} \cdot \tilde{\psi})\left(x K_{i}\right) & =\tilde{\phi} \otimes \tilde{\psi}\left(\Delta\left(x K_{i}\right)\right) \\
& =\sum_{(x)} \tilde{\phi}\left(x_{(1)} K_{i}\right) \tilde{\psi}\left(x_{(2)} K_{i}\right) \\
& =v^{\left(\alpha_{i}, w_{0}(\lambda+\mu)\right)} \sum_{(x)} \tilde{\phi}\left(x_{(1)}^{\prime} \prod K_{i}^{-a_{i}}\right) \tilde{\psi}\left(x_{(2)}\right) \\
& =v^{\left(\alpha_{i}, w_{0}(\lambda+\mu)\right)} v^{\left(-\beta, w_{0} \lambda\right)} \sum_{\operatorname{wt}\left(x_{(2)}\right)=\beta} \bar{\phi}\left(x_{(1)}^{\prime}\right) \bar{\psi}\left(x_{(2)}\right) \\
& =v^{\left(\alpha_{i}, w_{0}(\lambda+\mu)\right)} v^{\left(-\beta, w_{0} \lambda\right)}(\bar{\phi} \cdot \bar{\psi})(x) .
\end{aligned}
$$


7.2.7 It follows from 7.2.4 and Lemma 32 that if $q_{\lambda, \mu}$ denotes the homomorphism of $U_{v}(g)$-modules

$$
q_{\lambda, \mu}: V(\lambda) \otimes V(\mu) \simeq V(\lambda)^{*} \otimes V(\mu)^{*} \stackrel{i_{\lambda, \mu}^{*}}{\longrightarrow} V(\lambda+\mu)^{*} \simeq V(\lambda+\mu),
$$

then for $y \in V(\lambda)$ and $z \in V(\mu)$,

$$
\psi_{\lambda+\mu}\left(q_{\lambda, \mu}(y \otimes z)\right)=v^{\left(w_{0} \lambda,-\operatorname{wt}(z)+w_{0} \mu\right)} \psi_{\lambda}(y) \cdot \psi_{\mu}(z) .
$$

In particular, taking $t \in \operatorname{Tab}(\lambda), s \in \operatorname{Tab}(\mu)$ and $y=G^{*}(t), z=G^{*}(s)$, by [7.2.3 we have $\psi_{\lambda}(y)=G^{*}(\mathbf{m}), \psi_{\mu}(z)=G^{*}(\mathbf{n})$ for certain $\mathbf{m}, \mathbf{n} \in \mathcal{M}_{N}$, and

$$
\psi_{\lambda+\mu}\left(q_{\lambda, \mu}\left(G^{*}(t) \otimes G^{*}(s)\right)\right)=v^{-\left(w_{0} \lambda, \operatorname{wt}(\mathbf{n})\right)} G^{*}(\mathbf{m}) G^{*}(\mathbf{n}) .
$$

\subsection{We now come to the proof of Proposition 27}

7.3.1 Let $(L(\lambda), B(\lambda))$ be the upper crystal basis of $V(\lambda)$ Kas1, Kas2, Kas3. We recall that the upper crystal lattice $L(\lambda)$ is the $\mathbf{A}$-span of $\left\{G^{*}(t) \mid t \in \operatorname{Tab}(\lambda)\right\}$, and that $B(\lambda)$ is the $\mathbf{Q}$-basis $\left\{G^{*}(t) \bmod v L(\lambda) \mid t \in \operatorname{Tab}(\lambda)\right\}$ of the $\mathbf{Q}$-vector space $L(\lambda) / v L(\lambda)$. The elements of $B(\lambda)$ can be seen as combinatorial labels for the vectors of the (dual) canonical basis. We shall identify them with Young tableaux by writing $t$ in place of $G^{*}(t) \bmod v L(\lambda)$.

7.3.2 The comultiplication $\Delta^{\prime}$ of (15), (16), (17) is compatible with upper crystal bases Kas1, therefore $q_{\lambda, \mu}(L(\lambda) \otimes L(\mu))=L(\lambda+\mu)$. Moreover, if $t \otimes s$ belongs to the unique connected component of the crystal graph of $V(\lambda) \otimes V(\mu)$ isomorphic to $B(\lambda+\mu)$, then

$$
q_{\lambda, \mu}\left(G^{*}(t) \otimes G^{*}(s)\right) \equiv G^{*}(t \cdot s) \quad \bmod v L(\lambda+\mu)
$$

for some element $t \cdot s$ of $B(\lambda+\mu)=\operatorname{Tab}(\lambda+\mu)$.

7.3.3 For $t \in \operatorname{Tab}(\lambda)$, let $w(t)$ be the word obtained by reading the columns of $t$ in decreasing order and from left to right. Thus, if

$$
t=\begin{array}{|l|l|}
\hline 6 & \\
\hline 4 & 5 \\
\hline 2 & 3 \\
\hline 1 & 1 \\
\hline
\end{array}
$$

then $w(t)=6421531$. Recall the Robinson-Schensted map $w \mapsto(P(w), Q(w)) 6.1$ It is known (see [LT] ) that for $t \in \operatorname{Tab}(\lambda), s \in \operatorname{Tab}(\mu)$, the product $t \otimes s$ belongs to the connected component of the crystal of $V(\lambda) \otimes V(\mu)$ of type $B(\lambda+\mu)$ if and only if the concatenation $w(t) w(s)$ of the words $w(t)$ and $w(s)$ is mapped under the RobinsonSchensted map to a Young tableau $P(w(t) w(s))$ of shape $\lambda+\mu$. In this case $t \cdot s=$ $P(w(t) w(s))$. 
7.3.4 Using [7.2.7] we can now prove

Proposition 33 Let $\mathbf{m}, \mathbf{n} \in \mathcal{M}_{N}$. Suppose there exist $t \in \operatorname{Tab}(\lambda), s \in \operatorname{Tab}(\mu)$ such that $\mathbf{m}=\mathbf{m}(t), \mathbf{n}=\mathbf{m}(s)$ and $t \otimes s$ belongs to the connected component of $B(\lambda) \otimes B(\mu)$ of type $B(\lambda+\mu)$. Then

$$
v^{-\left(w_{0} \lambda, \operatorname{wt}(\mathbf{n})\right)} G^{*}(\mathbf{m}) G^{*}(\mathbf{n}) \equiv G^{*}(\mathbf{p}) \quad \bmod v \mathcal{L}^{*},
$$

where $\mathbf{p}=\mathbf{m}(t \cdot s)$.

Proof - By (18) we know that

$$
v^{-\left(w_{0} \lambda, \operatorname{wt}(\mathbf{n})\right)} G^{*}(\mathbf{m}) G^{*}(\mathbf{n})=\psi_{\lambda+\mu}\left(q_{\lambda, \mu}\left(G^{*}(t) \otimes G^{*}(s)\right)\right) .
$$

By [7.3.2 $q_{\lambda, \mu}\left(G^{*}(t) \otimes G^{*}(s)\right) \equiv G^{*}(t \cdot s) \bmod v L(\lambda+\mu)$. Since for $u \in \operatorname{Tab}(\lambda+\mu)$, by 7.2.3 $\psi_{\lambda+\mu}\left(G^{*}(u)\right)=G^{*}(\mathbf{m}(u))$, we deduce that

$$
\psi_{\lambda+\mu}\left(q_{\lambda, \mu}\left(G^{*}(t) \otimes G^{*}(s)\right)\right) \equiv G^{*}(\mathbf{m}(t \cdot s)) \quad \bmod v L^{*},
$$

where $L^{*}$ denotes the A-lattice spanned by the dual canonical basis of $U_{v}(\mathfrak{n})$. Hence we have proved

$$
v^{-\left(w_{0} \lambda, \operatorname{wt}(\mathbf{n})\right)} G^{*}(\mathbf{m}) G^{*}(\mathbf{n}) \equiv G^{*}(\mathbf{p}) \quad \bmod v L^{*} .
$$

On the other hand we know that $G^{*}(\mathbf{m}) G^{*}(\mathbf{n})$ is a $\mathbf{Z}\left[v, v^{-1}\right]$-linear combination of vectors of the dual canonical basis. Therefore the congruence is indeed modulo $v \mathcal{L}^{*}$. (Recall that $\mathcal{L}^{*}$ is the $\mathbf{Z}[v]$-lattice spanned by the dual canonical basis.)

7.3.5 Suppose now that $G^{*}(\mathbf{m})$ and $G^{*}(\mathbf{n})$ are quantum flag minors, namely, that $\mathbf{m}=$ $\mathbf{m}_{1}, \mathbf{n}=\mathbf{m}_{2}$ are associated with subsets $I_{1}, I_{2}$ of cardinality $n_{1}, n_{2}$ in the notation of 6.1. Then $G^{*}\left(\mathbf{m}_{1}\right)=\psi_{\Lambda_{N-n_{1}}}\left(G^{*}\left(t_{1}\right)\right), G^{*}\left(\mathbf{m}_{2}\right)=\psi_{\Lambda_{N-n_{2}}}\left(G^{*}\left(t_{2}\right)\right)$, where $t_{1}, t_{2}$ are the Young tableaux of column shape associated with the sets $[1, N] \backslash I_{1},[1, N] \backslash I_{2}$. Applying Proposition 33 in this case, we see that we obtain precisely Proposition [27] for the product of two quantum flag minors.

7.3.6 The extension to a product of $r>2$ minors is straightforward. First, [7.2.7 generalizes easily as follows. If $t_{i} \in \operatorname{Tab}\left(\lambda^{(i)}\right)$ and $\psi_{\lambda^{(i)}}\left(G^{*}\left(t_{i}\right)\right)=G^{*}\left(\mathbf{m}_{i}\right)(i=1, \ldots, r)$, then, denoting by $q_{\lambda^{(1)}, \ldots, \lambda^{(r)}}$ the projection $V\left(\lambda^{(1)}\right) \otimes \cdots \otimes V\left(\lambda^{(r)}\right) \longrightarrow V\left(\lambda^{(1)}+\cdots+\lambda^{(r)}\right)$ we have

$\psi_{\lambda^{(1)}+\cdots+\lambda^{(r)}}\left(q_{\lambda^{(1)}, \ldots, \lambda^{(r)}}\left(G^{*}\left(t_{1}\right) \otimes \cdots \otimes G^{*}\left(t_{r}\right)\right)\right)=v^{-\sum_{i<j}\left(w_{0} \lambda^{(i)}, \operatorname{wt}\left(\mathbf{m}_{j}\right)\right)} G^{*}\left(\mathbf{m}_{1}\right) \cdots G^{*}\left(\mathbf{m}_{r}\right)$.

Secondly, 7.3 .2 and 7.3 .3 generalize in the obvious way, namely, if $t_{1} \otimes \cdots \otimes t_{r}$ belongs to the connected component of $B\left(\lambda^{(1)}\right) \otimes \cdots \otimes B\left(\lambda^{(r)}\right)$ of type $B\left(\lambda^{(1)}+\cdots \lambda^{(r)}\right)$, then

$$
q_{\lambda^{(1)}, \ldots, \lambda^{(r)}}\left(G^{*}\left(t_{1}\right) \otimes \cdots \otimes G^{*}\left(t_{r}\right)\right) \equiv G^{*}\left(t_{1} \cdots t_{r}\right) \quad \bmod v L\left(\lambda^{(1)}+\cdots+\lambda^{(r)}\right)
$$

where $t_{1} \cdots t_{r}=P\left(w\left(t_{1}\right) \cdots w\left(t_{r}\right)\right)$. From these facts one obtains easily the generalization of Proposition 33 to $r$ factors, and by specializing to quantum flag minors one gets the general case of Proposition 27. This finishes the proof of Proposition 27]

7.4 In this section we prove Theorem 30 
7.4.1 Let us recall the realization of the finite-dimensional irreducible $U_{v}(\mathfrak{g})$-modules $V(\lambda)$ as subspaces of the deformation $A_{v}[\mathcal{F}]$ of the coordinate ring of the flag variety [TT, LT, LZ]. The algebra $A_{v}[\mathcal{F}]$ is generated over $\mathbf{Q}(v)$ by $2^{N}-1$ generators $[J]$ labelled by the non-empty subsets of $[1, N]$ submitted to the relations $R_{1}$ and $R_{2}$ below. Given two subsets $A$ and $B$ of $[1, N]$, write $\operatorname{inv}(A, B)$ for the number of pairs $(a, b) \in A \times B$ with $a>b$.

$R_{1}(I, J)$ : for all subsets $I$ and $J$ such that $|I| \leqslant|J|$, we have

$$
[I][J]=\sum_{M}(-v)^{\operatorname{inv}(J \backslash M, M)-\operatorname{inv}(I, M)}[I \cup M][J \backslash M],
$$

where the sum is over all $M \subset J \backslash I$ with $|M|=|J|-|I|$;

$R_{2}(I, J)$ : for all subsets $I$ and $J$ such that $|I|-1 \geqslant|J|+1$, we have

$$
\sum_{i \in I \backslash J}(-v)^{\operatorname{inv}(\{i\}, I \backslash\{i\})-\operatorname{inv}(\{i\}, J)}[I \backslash\{i\}][J \cup\{i\}]=0 .
$$

To each subset $I$ of cardinality $r \leqslant N$ we associate the vector $G^{*}(I)$ of the fundamental representation $V\left(\Lambda_{r}\right)$. Let $\left(i_{1}, \ldots, i_{r}\right) \in[1, N-1]^{r}$ and set $\lambda=\sum_{k=1}^{r} \Lambda_{i_{k}}$. (Here, as in 7.2.3 we identify $I \subset[1, N]$ to a column Young tableau.) Define a linear map $Q_{\Lambda_{i_{1}}, \ldots, \Lambda_{i_{r}}}$ from $V\left(\Lambda_{i_{1}}\right) \otimes \cdots \otimes V\left(\Lambda_{i_{r}}\right)$ to $A_{v}[\mathcal{F}]$ by

$$
Q_{\Lambda_{i_{1}}, \ldots, \Lambda_{i_{r}}}\left(G^{*}\left(I_{1}\right) \otimes \cdots \otimes G^{*}\left(I_{r}\right)\right)=\left[I_{1}\right] \cdots\left[I_{r}\right] .
$$

Then $\operatorname{ker}\left(Q_{\Lambda_{i_{1}}, \ldots, \Lambda_{i_{r}}}\right)=\operatorname{ker}\left(q_{\Lambda_{i_{1}}, \ldots, \Lambda_{i_{r}}}\right)$, hence $Q_{\Lambda_{i_{1}}, \ldots, \Lambda_{i_{r}}}$ induces an embedding of $V(\lambda)$ in $A_{v}[\mathcal{F}]$ which maps $q_{\Lambda_{i_{1}}, \ldots, \Lambda_{i_{r}}}\left(G^{*}\left(I_{1}\right) \otimes \cdots \otimes G^{*}\left(I_{r}\right)\right)$ to $\left[I_{1}\right] \cdots\left[I_{r}\right]$. In other words the linear relations in $V(\lambda)$ between the vectors $q_{\Lambda_{i_{1}}, \ldots, \Lambda_{i_{r}}}\left(G^{*}\left(I_{1}\right) \otimes \cdots \otimes G^{*}\left(I_{r}\right)\right)$ are exactly the same as those in $A_{v}[\mathcal{F}]$ between the vectors $\left[I_{1}\right] \cdots\left[I_{r}\right]$.

7.4.2 Let $I$ and $J$ be two separated subsets of $[1, N]$ and write $I_{0}=I \backslash(I \cap J), J_{0}=$ $J \backslash(I \cap J)$. Up to a possible exchange of $I$ and $J$, we can assume that $|I| \leqslant|J|$ and $I_{0}=I^{\prime} \cup I^{\prime \prime}$ with $I^{\prime} \prec J_{0} \prec I^{\prime \prime}$.

Proposition 34 The following relation holds in $A_{v}[\mathcal{F}]$ :

$$
[J][I]=v^{\left|I^{\prime}\right|} \sum_{M}(-v)^{\operatorname{inv}\left(M, J_{0} \backslash M\right)}\left[I^{\prime} \cup(J \backslash M)\right]\left[M \cup\left(I \backslash I^{\prime}\right)\right]
$$

where the sum is over all $M \subset J_{0}$ with $|M|=\left|I^{\prime}\right|$.

Proof - First, because of $[\mathbf{L Z}]$ Lemma 2.3, it is enough to prove the proposition in the case $I \cap J=\emptyset$, so we can assume that $I_{0}=I$ and $J_{0}=J$. As a second reduction, we remark that the subset $I^{\prime \prime}$ is contained in all right factors of the identity, and that we have $I^{\prime \prime} \succ I^{\prime}$ and $I^{\prime \prime} \succ M$. Thus, by using the $v$-analogue of Laplace's expansion ( [T T Proposition 2.10) we may erase $I^{\prime \prime}$ in all terms and we are reduced to prove

$$
[J]\left[I^{\prime}\right]=v^{\left|I^{\prime}\right|} \sum_{M}(-v)^{\operatorname{inv}(M, J \backslash M)}\left[I^{\prime} \cup(J \backslash M)\right][M] .
$$


Now since $I^{\prime} \prec J$ and $\left|I^{\prime}\right| \leqslant|J|$ we have $[J]\left[I^{\prime}\right]=v^{\left|I^{\prime}\right|}\left[I^{\prime}\right][J]$ and the identity to be proved becomes

$$
\left[I^{\prime}\right][J]=\sum_{M}(-v)^{\operatorname{inv}(M, J \backslash M)}\left[I^{\prime} \cup(J \backslash M)\right][M] .
$$

Writing $M^{\prime}=J \backslash M$, we can rewrite it as

$$
\left[I^{\prime}\right][J]=\sum_{M^{\prime}}(-v)^{\operatorname{inv}\left(J \backslash M^{\prime}, M^{\prime}\right)}\left[I^{\prime} \cup M^{\prime}\right]\left[J \backslash M^{\prime}\right] .
$$

This is exactly $R_{1}\left(I^{\prime}, J\right)$, since $I^{\prime} \prec J \operatorname{implies} \operatorname{inv}\left(I^{\prime}, M^{\prime}\right)=0$ for all $M^{\prime} \subset J$.

Example 35 In $A_{v}[\mathcal{F}]$ we have

$$
\begin{aligned}
{[3,4,5,6][1,2,7]=} & v^{2}[1,2,5,6][3,4,7]-v^{3}[1,2,4,6][3,5,7]+v^{4}[1,2,3,6][4,5,7] \\
& +v^{4}[1,2,4,5][3,6,7]-v^{5}[1,2,3,5][4,6,7]+v^{6}[1,2,3,4][5,6,7] .
\end{aligned}
$$

7.4.3 Write $|I|=i$ and $|J|=j$. Using [7.4.1 we identify $V\left(\Lambda_{i}+\Lambda_{j}\right)$ with the subspace spanned by the vectors $[K][L]$ with $|K|=j$ and $|L|=i$. We can then interpret (19) as a relation between the vectors $q_{\Lambda_{j}, \Lambda_{i}}\left(G^{*}(K) \otimes G^{*}(L)\right)$. Note that all the products $[K][L]$ occuring in the right-hand side of (19) have the property that if we arrange the elements of $K$ and $L$ in increasing order into two columns shape Young tableaux $t(K)$ and $t(L)$, the juxtaposition $t(K) t(L)$ is a semi-standard Young tableau $t(K, L)$. Therefore, by 7.3 .2 . 7.3.3, we have

$$
[K][L] \equiv G^{*}(t(K, L)) \quad \bmod v L\left(\Lambda_{j}+\Lambda_{i}\right) .
$$

Moreover, the term $[K][L]$ having the lowest power of $v$ is the one in which $M$ consists of the $\left|I^{\prime}\right|$ smaller elements of $J_{0}$, and the corresponding tableau $t_{\text {low }}$ is obtained by reordering the rows of the juxtaposition $t(I) t(J)$. It follows that, using the notation of $7.2 .3, \mathbf{m}\left(t_{\text {low }}\right)=$ $\mathbf{m}(t(I))+\mathbf{m}(t(J))$. Define

$$
\bar{I}=[1, N] \backslash I, \quad \bar{J}=[1, N] \backslash J, \quad \mathcal{I}=\mathbf{Z}_{\leqslant 0} \cup \bar{I}, \quad \mathcal{J}=\mathbf{Z}_{\leqslant 0} \cup \bar{J} .
$$

Then, by 7.2 .3

$$
\psi_{\Lambda_{i}}([I])=\langle\mathcal{I}\rangle=G^{*}(\mathbf{m}(t(I))), \quad \psi_{\Lambda_{j}}([J])=\langle\mathcal{J}\rangle=G^{*}(\mathbf{m}(t(J)))
$$

and

$$
\psi_{\Lambda_{j}+\Lambda_{i}}\left(G^{*}\left(t_{\text {low }}\right)\right)=G^{*}(\mathbf{m}(t(I))+\mathbf{m}(t(J))) .
$$

Hence, applying $\psi_{\Lambda_{j}+\Lambda_{i}}$ to both sides of (19) we get

$$
v^{k}\langle\mathcal{J}\rangle\langle\mathcal{I}\rangle=v^{k} G^{*}(\mathbf{m}(t(J))) G^{*}(\mathbf{m}(t(I))) \equiv G^{*}(\mathbf{m}(t(I))+\mathbf{m}(t(J))) \quad \bmod v \mathcal{L}^{*},
$$

for some integer $k$. Since we know that $G^{*}(\mathbf{m}(t(I))+\mathbf{m}(t(J)))$ occurs in $\langle\mathcal{J}\rangle\langle\mathcal{I}\rangle$ with coefficient $v^{-b(\mathbf{m}(t(J)), \mathbf{m}(t(I)))}$, we see that $k=b(\mathbf{m}(t(J)), \mathbf{m}(t(I)))$. Note that $I$ and $J$ are separated if and only if $\bar{I}$ and $\bar{J}$ are separated. Thus, using the reformulation of the Berenstein-Zelevinsky conjecture given by Conjecture 22, we have obtained that if $\mathcal{I}$ and $\mathcal{J}$ are separated, $v^{b(\mathbf{m}(t(J)), \mathbf{m}(t(I)))}\langle\mathcal{J}\rangle\langle\mathcal{I}\rangle$ belongs to the dual canonical basis $\mathcal{B}_{v}$. This finishes the proof of Theorem 30 . 


\section{$8 \quad$ Irreducibility of induction products of evaluation modules}

8.1 Let $a$ be an integer, $\alpha=\left(\alpha_{1}, \ldots, \alpha_{r}\right)$ a partition of $m$, and let $S_{\alpha}\left(t^{a}\right)$ denote the corresponding evaluation module of the affine Hecke algebra $\widehat{H}_{m}(t)$. Recall from 2.4 that $S_{\alpha}\left(t^{a}\right) \simeq L_{\mathbf{m}(\alpha, a)}$, where $\mathbf{m}(\alpha, a)=\sum_{i=1}^{r}\left[a-i+1, a-i+\alpha_{i}\right]$. Therefore, by Theorem [12, the class of $S_{\alpha}\left(t^{a}\right)$ in the Grothendieck ring $R$ is mapped under the isomorphism $\Psi$ to the vector $g^{*}(\mathbf{m}(\alpha, a))$ of $A$. By Proposition 24, the corresponding element of $A_{v}$ is

$$
G^{*}(\mathbf{m}(\alpha, a))=\Delta\left([a-r+1, a],\left\{a-r+1+\alpha_{r}, \ldots, a+\alpha_{1}\right\}\right)
$$

that is, a quantum flag minor. It follows that we can apply Proposition 25. Theorem 13 and Theorem 30 and get a completely explicit answer to the problem of the irreducibility of the induction product of two evaluation modules.

Let $\beta=\left(\beta_{1}, \ldots, \beta_{s}\right)$ be another partition, and let $b \in \mathbf{Z}$. We set

$$
\mathcal{I}:=\mathbf{Z}_{\leqslant a-r} \cup\left\{a-r+1+\alpha_{r}, \ldots, a+\alpha_{1}\right\}, \quad \mathcal{J}:=\mathbf{Z}_{\leqslant b-s} \cup\left\{b-s+1+\beta_{s}, \ldots, b+\beta_{1}\right\} .
$$

Then we have $G^{*}(\mathbf{m}(\alpha, a))=\langle\mathcal{I}\rangle$ and $G^{*}(\mathbf{m}(\beta, b))=\langle\mathcal{J}\rangle$ in the notation of Section [5] Hence, we get

Theorem 36 The induction product $S_{\alpha}\left(t^{a}\right) \odot S_{\beta}\left(t^{b}\right)$ is irreducible if and only if the sets $\mathcal{I}$ and $\mathcal{J}$ are separated.

Example 37 Take $\alpha=(4,2)$ and $\beta=(2,2,1)$. We want to determine for which values of $a$ and $b$ the induction product $S_{\alpha}\left(t^{a}\right) \odot S_{\beta}\left(t^{b}\right)$ is irreducible. Clearly, this only depends on the difference $b-a$ and we can take $a=0$. We have

$$
\mathcal{I}=\mathbf{Z}_{\leqslant-2} \cup\{1,4\}, \quad \mathcal{J}=\mathbf{Z}_{\leqslant b-3} \cup\{b-1, b+1, b+2\} .
$$

For $b \leqslant-4$ we have $\mathcal{J} \subset \mathcal{I}$, and for $b \geqslant 7, \mathcal{I} \subset \mathcal{J}$. Thus if $b \notin[-3,6], \mathcal{I}$ and $\mathcal{J}$ are trivially separated and the product is irreducible. For the remaining values we find that $\mathcal{I}$ and $\mathcal{J}$ are separated for $b=0$, strongly separated for $b=2,5$, and not separated for $b=-3,-2,-1,1,3,4,6$. Hence $S_{\alpha}\left(t^{a}\right) \odot S_{\beta}\left(t^{b}\right)$ is simple if and only if $b-a \notin$ $\{-3,-2,-1,1,3,4,6\}$.

It is easy to deduce from the definition of separated sets given in 5.2 that Theorem 36 is equivalent to the statement of Theorem 1 in the Introduction. Let us check it. We first note that the sets $\mathcal{I} \backslash \mathcal{J}$ and $\mathcal{J} \backslash \mathcal{I}$ are finite and that we have the following relation between their cardinalities: $|\mathcal{I} \backslash \mathcal{J}|=|\mathcal{J} \backslash \mathcal{I}|+a-b$. Therefore, if $a-b>0$, then $|\mathcal{I} \backslash \mathcal{J}|>|\mathcal{J} \backslash \mathcal{I}|$ and the sets $\mathcal{I}$ and $\mathcal{J}$ are not separated if and only if there exist $i, j, k$ such that $i, k \in \mathcal{I} \backslash \mathcal{J}$, $j \in \mathcal{J} \backslash \mathcal{I}$ and $i<j<k$ (see $[\mathbf{L Z}, 3.8$ ). The case $a-b<0$ is similar. If $a=b$, the two sets $\mathcal{I} \backslash \mathcal{J}$ and $\mathcal{J} \backslash \mathcal{I}$ being of equal cardinality, they are not separated if and only if there exist $i, j, k, l$ such that $i, k \in \mathcal{I} \backslash \mathcal{J}, j, l \in \mathcal{J} \backslash \mathcal{I}$ and either $i<j<k<l$ or $j<i<l<k$ ([LZ, 3.8$)$.

We note the following obvious corollary of Theorem 1 which will be used in the proof of Theorem 2 .

Corollary 38 Let $L=S_{\alpha}(z)$ be an evaluation module of $\widehat{H}_{m}$. Then, $L \odot L$ is irreducible. 


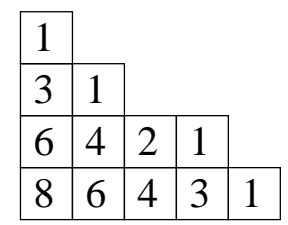

Figure 2: The hook-lengths of $\alpha=(5,4,2,1)$

The criterion of irreducibility takes an especially nice form when $\alpha=\beta$. Let $\alpha^{\prime}=$ $\left(d_{1}, \ldots, d_{k}\right)$ denote the conjugate partition. Recall that the hook-length of the cell $c_{i j}$ of the Young diagram of $\alpha$ lying on row $i$ and column $j$ is defined by

$$
h_{i j}=\alpha_{i}+d_{j}-i-j+1 .
$$

This is illustrated in Figure 2 .

Corollary 39 The induction product $S_{\alpha}\left(t^{a}\right) \odot S_{\alpha}\left(t^{b}\right)$ is irreducible if and only if $|b-a|$ is not one of the hook-lengths of $\alpha$.

Proof - Since $\alpha=\beta$, the sets $\mathcal{I}$ and $\mathcal{J}$ simply differ by a translation of $b-a$. Let $\mathcal{I}_{0}:=\mathcal{I} \backslash(\mathcal{I} \cap \mathcal{J})$ and $\mathcal{J}_{0}:=\mathcal{J} \backslash(\mathcal{I} \cap \mathcal{J})$. If $b-a>0$ then $\left|\mathcal{J}_{0}\right|>\left|\mathcal{I}_{0}\right|, a+r+1, b+\alpha_{1}$ belong to $\mathcal{J}_{0}$ and $\left.\mathcal{I}_{0} \subset\right] a+r+1, b+\alpha_{1}$. Therefore $\mathcal{I}$ and $\mathcal{J}$ are separated if and only if $\mathcal{I}_{0}=\emptyset$, and in this case, they are strongly separated. It is now a simple exercise to verify that $\mathcal{I} \subset \mathcal{J}$ if and only if $b-a$ is not one of the hook-lengths of $\alpha$.

8.2 Our proof of Theorem 1 was based on the theory of canonical bases for the quantum algebras $U_{v}\left(\mathfrak{s} l_{N}\right)$. There, $v$ was regarded as a formal variable. To prove Theorem 2 we will employ the representation theory of the quantum enveloping algebras $U_{v}\left(\widehat{\mathfrak{s l}}_{N}\right)$ of the Kac-Moody Lie algebras $\widehat{\mathfrak{s l}}_{N}$. But in this proof we will set $v=t^{\frac{1}{2}}$.

8.2.1 The link between the representation theories of the affine Hecke algebras $\widehat{H}_{m}=$ $\widehat{H}_{m}(t)$ and the quantum enveloping algebras $U_{v}\left(\widehat{\mathfrak{s}}_{N}\right)$ was discovered by Drinfeld $[\mathbf{D}$, in the degenerate case. In the non-degenerate case this link was established by Cherednik in Ch. We will use the version of this link due to Chari and Pressley CP. In particular, we will take the definition of the Hopf algebra $U_{v}\left(\widehat{\mathfrak{s l}}_{N}\right)$ from $\mathbf{C P}, 2.1$ and 2.4. Note that $U_{v}\left(\widehat{\mathfrak{s l}}_{N}\right)$ contains $U_{v}\left(\mathfrak{s} l_{N}\right)$ as a Hopf subalgebra. Our definition of the affine Hecke algebra $\widehat{H}_{m}$ coincides with the definition from [CP], 3.1. Fix the integer $N \geqslant 2$. Set $v=t^{\frac{1}{2}}$.

For each $m=1,2, \ldots$ there is a functor $\mathcal{D}$ from the category of all finite-dimensional $\widehat{H}_{m}$-modules to the category of finite-dimensional $U_{v}\left(\widehat{\mathfrak{s l}}{ }_{N}\right)$-modules which are of level $m$ as $U_{v}\left(\mathfrak{s} l_{N}\right)$-modules $(\mathbf{C P}, 4.2)$. Recall that a $U_{v}\left(\mathfrak{s} l_{N}\right)$-module is said to be of level $m$ if all its irreducible components occur in the $m$ th tensor power of the vector representation of $U_{v}\left(\mathfrak{s} l_{N}\right)$.) Moreover, under the assumption $N>m$, the functor $\mathcal{D}$ is an equivalence of categories. The proofs of all these statements have been given in [CP.

Denote by $V_{\alpha}(z)$ the image under the functor $\mathcal{D}$ of the evaluation $\widehat{H}_{m}$-module $S_{\alpha}(z)$. This is an irreducible module over the quantum affine algebra $U_{v}\left(\widehat{\mathfrak{s} l} l_{N}\right)$, which is also called an evaluation module $(\underline{\mathbf{C P}}, 5.4)$. Consider also the image $V_{\beta}(w)$ of the $\widehat{H}_{n}$-module $S_{\beta}(w)$. 
Suppose that $N>m, n$. Then the modules $V_{\alpha}(z), V_{\beta}(w)$ are non-zero. By $\mathbf{C P}, 4.4$ the $U_{v}\left(\widehat{\mathfrak{s l}}_{N}\right)$-module $\mathcal{D}\left(S_{\alpha}(z) \odot S_{\beta}(w)\right)$ is equivalent to the tensor product $V_{\alpha}(z) \otimes V_{\beta}(w)$.

By [Ze1], 8.7 the induction products $S_{\alpha}(z) \odot S_{\beta}(w)$ and $S_{\beta}(w) \odot S_{\alpha}(z)$ are irreducible and equivalent whenever the ratio $z / w$ does not belong to $t^{\mathbf{Z}}=v^{2 \mathbf{Z}}$. Then the $U_{v}\left(\widehat{\mathfrak{s l}}_{N}\right)$ modules $V_{\alpha}(z) \otimes V_{\beta}(w)$ and $V_{\beta}(w) \otimes V_{\alpha}(z)$ are also irreducible and equivalent. So then there exists a non-zero $U_{v}\left(\widehat{\mathfrak{s l}}_{n}\right)$-intertwining operator

$$
I_{\alpha \beta}(z, w): V_{\alpha}(z) \otimes V_{\beta}(w) \longrightarrow V_{\beta}(w) \otimes V_{\alpha}(z),
$$

unique up to a multiplier from $\mathbf{C}^{*}$. By definition, the vector spaces of the modules $V_{\alpha}(z)$ are the same for different values of the parameter $z$, and so are the spaces of the modules $V_{\beta}(w)$ for different values of $w$. The multipliers from $\mathbf{C}^{*}$ can be so chosen that the linear operator $I_{\alpha \beta}(z, w)$ depends on $z, w$ as a rational function of $z / w$. Such a choice can be made by using the explicit realizations of the modules $V_{\alpha}(z)$ and $V_{\beta}(w)$ from $[\mathbf{C h}]$, Proposition 1.5. Assume that such a choice has been made, and write $I_{\alpha \beta}(z, w)=I_{\alpha \beta}(z / w)$. In the physical literature the operator $I_{\alpha \beta}(z / w)$ is called the trigonometric $\check{R}$-matrix corresponding to the evaluation modules $V_{\alpha}(z)$ and $V_{\beta}(w)$, see for instance [KMT, $\mathbf{M T}$.

8.2.2 When $N>m+n$, the irreducibility of the $U_{v}\left(\widehat{\mathfrak{s l}}_{N}\right)$-module $V_{\alpha}(z) \otimes V_{\beta}(w)$ is equivalent to the irreducibility of the $\widehat{H}_{m+n}$-module $S_{\alpha}(z) \odot S_{\beta}(w)$. Thus for $N>m+n$ the module $V_{\alpha}(z) \otimes V_{\beta}(w)$ is reducible, if and only if $z / w=t^{c}$ for $c \in \mathbf{Z}$ described by our Theorem 1. For an arbitrary $N$, an irreducibility criterion for $V_{\alpha}(z) \otimes V_{\beta}(w)$ has been recently given by Molev in $[\mathbf{M}]$, independently of our results and by different methods; see Theorems 3.1 and 4.1 therein. Actually, Molev considers representations of the Yangian of $\mathfrak{g} l_{N}$, but it is known that the finite dimensional representation theory of the quantized affine algebra and that of the Yangian coincide, see $\mathbf{V a}$.

8.2.3 Proof of Theorem 2, For each $k=1, \ldots, r$ consider the evaluation module $S_{\alpha^{(k)}}\left(z_{k}\right)$. This is an irreducible module over the affine Hecke algebra $\widehat{H}_{m_{k}}$ where $\alpha^{(k)}$ is a partition of $m_{k}$. Take the irreducible $U_{v}\left(\widehat{\mathfrak{s}}_{N}\right)$-module $V_{k}=V_{\alpha(k)}\left(z_{k}\right)$. Suppose $N>m_{k}$ for every $k$, so that $V_{k}$ is not zero. The $U_{v}\left(\widehat{\mathfrak{s} l} N_{N}\right)$-module $\mathcal{D}\left(S_{\alpha^{(1)}}\left(z_{1}\right) \odot \ldots \odot S_{\alpha^{(r)}}\left(z_{r}\right)\right)$ is equivalent to the tensor product

$$
\mathcal{D}\left(S_{\alpha^{(1)}}\left(z_{1}\right)\right) \otimes \ldots \otimes \mathcal{D}\left(S_{\alpha^{(r)}}\left(z_{r}\right)\right)=V_{1} \otimes \ldots \otimes V_{r} .
$$

We will demonstrate that the $U_{v}\left(\widehat{\mathfrak{s l}}_{N}\right)$-module $V_{1} \otimes \ldots \otimes V_{r}$ is irreducible, if and only if the tensor products $V_{k} \otimes V_{l}$ are irreducible for all $k<l$. When we choose $N>m_{1}+\ldots+m_{r}$ here, Theorem 2 will follow.

Let $k, l=1, \ldots, r$. By taking the first non-zero coefficient of the Laurent expansion of the rational function $I_{\alpha^{(k)} \alpha^{(l)}}(u)$ at $u \rightarrow z_{k} / z_{l}$, we obtain an $U_{v}\left(\widehat{\mathfrak{s}}_{N}\right)$-intertwining operator $I_{k l}: V_{k} \otimes V_{l} \longrightarrow V_{l} \otimes V_{k}$. By Corollary 38 the $\widehat{H}_{2 m_{k}}$-module $S_{\alpha^{(k)}}\left(z^{(k)}\right) \odot S_{\alpha^{(k)}}\left(z^{(k)}\right)$ is irreducible for every $k=1, \ldots, r$. So the tensor square $V_{k} \otimes V_{k}$ is also irreducible. This implies that $I_{k k}$ equals the unit operator in $V_{k} \otimes V_{k}$, up to a multiplier. A different proof of this equality has been given in DJKM]. Under these unit conditions the argument of KMT, MT shows, that the irreducibility of the tensor product $V_{1} \otimes \ldots \otimes V_{r}$ is equivalent to the invertibility of all operators $I_{k l}$ with $k<l$. Here we again use the explicit realizations of the modules $V_{k}$ from [Ch], Proposition 1.5. For the details of this argument see [NT2]. Applying this result in the particular case $r=2$, we obtain that the invertibility of the 
single operator $I_{k l}$ with $k<l$ is equivalent to the irreducibility of the tensor product $V_{k} \otimes V_{l}$. This completes the proof of Theorem 2 ,

8.2.4 We note that in the case where all the partitions $\alpha^{(k)}$ are rectangular, the irreducibility criterion for the module $V_{1} \otimes \ldots \otimes V_{r}$ was obtained in [NT1. In particular, it was shown in [NT1] that for the rectangular partitions $\alpha^{(k)}$, the module $V_{1} \otimes \ldots \otimes V_{r}$ is irreducible, if and only if the pairwise tensor products $V_{k} \otimes V_{l}$ are irreducible for all $k<l$. In fact, NT1 discusses representations of the Yangian of $\mathfrak{g} l_{N}$, but as mentioned above, the finite dimensional representation theory of $U_{v}\left(\widehat{\mathfrak{s} l} N_{N}\right)$ and that of the Yangian coincide.

8.2.5 Let us make an important remark on the trigonometric $\check{R}$-matrix $I_{\alpha \beta}(u)$. This is an operator-valued rational function of $u$, determined by two partitions $\alpha$ and $\beta$ of $m$ and $n$ respectively. It also depends on the choice of $N>m, n$. By definition, all zeroes and poles of this rational function of $u$ belong to $t^{\mathbf{Z}}$. Let us call a point $c \in \mathbf{Z}$ singular, if the first non-zero coefficient in the Laurent expansion of $I_{\alpha \beta}(u)$ at $u \rightarrow t^{c}$ is a non-invertible linear operator. Our proof of Theorem 2 shows, that for $N>m+n$ the point $c \in \mathbf{Z}$ is singular, if and only if the induced module $S_{\alpha}(z) \odot S_{\beta}(w)$ with $z / w=t^{c}$ is reducible. Thus when $N>m+n$, the list of all singular points $c$ for $I_{\alpha \beta}(u)$ is also given by Theorem 1 In the particular case when every part of the partitions $\alpha$ and $\beta$ is 1 , this result follows from $\mathbf{A K}$.

\subsection{Proof of Theorem 31. Consider the product}

$$
\pi=\left\langle\mathcal{I}_{1}\right\rangle \cdots\left\langle\mathcal{I}_{r}\right\rangle=G^{*}\left(\mathbf{m}_{1}\right) \cdots G^{*}\left(\mathbf{m}_{r}\right)
$$

of $r$ quantum flag minors. By Theorem 12 and Proposition [15] the normalized product $v^{b} \pi$ belongs for some $b \in \mathbf{Z}$ to the canonical basis $\mathcal{B}_{v}$ if and only if the induction product $L=L_{\mathbf{m}_{1}} \odot \cdots \odot L_{\mathbf{m}_{r}}$ is irreducible. By Theorem 2, the irreducibility of $L$ is equivalent to the irreducibility of all pairwise induction products $L_{\mathbf{m}_{k}} \odot L_{\mathbf{m}_{l}}$ where $1 \leqslant k<l \leqslant r$.

Again by Theorem 12 and Proposition [15, the induction product $L_{\mathbf{m}_{k}} \odot L_{\mathbf{m}_{l}}$ is irreducible, if and only if the product of two quantum flag minors $G^{*}\left(\mathbf{m}_{k}\right) G^{*}\left(\mathbf{m}_{l}\right)=\left\langle\mathcal{I}_{k}\right\rangle\left\langle\mathcal{I}_{l}\right\rangle$ belongs to $\mathcal{B}_{v}$ up to a power of $v$. But by Theorem [30, this happens if and only the quantum flag minors $\left\langle\mathcal{I}_{k}\right\rangle$ and $\left\langle\mathcal{I}_{l}\right\rangle$ quasi-commute.

Acknowledgements. We would like to express our gratitude to N. Kitanine for drawing our attention to the results of [KMT, $\mathbf{M T}$. Support from the EPSRC and from the EC under the TMR grant FMRX-CT97-0100 is gratefully acknowledged.

\section{References}

[AK] T. Akasaka, M. Kashiwara, Finite-dimensional representations of quantum affine algebras, Publ. Res. Inst. Math. Sci. 33 (1997), 839-867.

[Ar] S. ARIKI, On the decomposition numbers of the Hecke algebra of $G(n, 1, m)$, J. Math. Kyoto Univ. 36 (1996), 789-808.

[BZ1] A. Berenstein, A. Zelevinsky, String bases for quantum groups of type $A_{r}$, Advances in Soviet Math. (Gelfand's seminar) 16 (1993), 51-89. 
[BZ2] A. Berenstein, A. Zelevinsky, Canonical bases for the quantum group of type $A_{r}$ and piecewise-linear combinatorics, Duke Math. J. 82 (1996), 473-502.

[CP] V. Chari, A. Pressley, Quantum affine algebras and affine Hecke algebras, Pacific J. Math. 174 (1996), 295-326.

[Ch] I. Cherednik, A new interpretation of Gel'fand-Tzetlin bases, Duke Math. J. 54 (1987), 563-577.

[CG] N. Chriss, V. GinzBurg, Representation theory and complex geometry, Birkhauser 1997.

[D] V. Drinfeld, Degenerate affine Hecke algebras and Yangians, Funct. Anal. Appl. 20 (1986), 56-58.

[DJKM] E. Date, M. Jimbo, A. Kuniba, T. Miwa, A quantum trace formula for R-matrices, in Selected topics in conformal field theory and statistical mechanics, Proceedings of the 8-th symposium on theoretical physics, Mt. Sorak 1989, H. S. Song, Ed., Kyohak Yunkusa, 1990, 87-111.

[Fu] W. Fulton, Young tableaux, L.M.S. Student Texts 35, Cambridge 1997.

[FL] W. Fulton, A. Lascoux, A Pieri formula in the Grothendieck ring of a flag bundle, Duke Math. J. 76 (1994), 711-729.

[Gi] V. GinzBurg, Geometric aspects of representation theory, in Proceedings of ICM 1986, Berkeley, 304308.

[Kas1] M. Kashiwara, Crystalizing the q-analogue of universal enveloping algebras, Commun. Math. Phys. 133 (1990), 249-260.

[Kas2] M. Kashiwara, On crystal bases of the q-analogue of universal enveloping algebras, Duke Math. J. 63 (1991), 465-516.

[Kas3] M. Kashiwara, Global crystal bases of quantum groups, Duke Math. J. 69 (1993), 455-485.

[Kat] S. KATO, Irreducibility of principal series representations for Hecke algebras of affine type, J. Fac. Sci. Univ. Tokyo 28 (1981), 929-943.

[KMT] N. Kitanine, J.-M. Maillet, V. Terras, Form factors of the XXZ Heisenberg spin- $\frac{1}{2}$ finite chain, Nuclear Phys. B 554 (1999), 647-678.

[LLT] A. Lascoux, B. Leclerc and J.-Y. Thibon, Hecke algebras at roots of unity and crystal bases of quantum affine algebras, Commun. Math. Phys. 181 (1996), 205-263.

[LS] A. Lascoux, M.P. Schützenberger, Keys and standard bases, in Invariant theory and tableaux, D. Stanton, Ed., IMA 19, Springer, 1990, 125-144.

[LZ] B. Leclerc, A. Zelevinsky, Quasicommuting families of quantum Plücker coordinates, Advances in Math. Sciences (Kirillov's seminar), AMS Translations 181 (1998), 85-108.

[LT] B. Leclerc, J.-Y. Thibon, The Robinson-Schensted correspondence, crystal bases, and the quantum straightening at $q=0$, Elect. J. Alg. Combinatorics 3 (1996), R11.

[LTV] B. Leclerc, J.-Y. Thibon, E. Vasserot, Zelevinsky's involution at roots of unity, J. Reine Angew. Math. 513 (1999), 33-51.

[Lu1] G. Lusztig, Canonical bases arising from quantized enveloping algebras, I, J. Amer. Math. Soc. 3 (1990), 447-498.

[Lu2] G. LuszTig, Quivers, perverse sheaves and quantized enveloping algebras, J. Amer. Math. Soc. 4 (1991), $365-421$.

[MT] J.-M. Maillet, V. Terras, On the quantum inverse scattering problem, Nuclear Phys. B 575 (2000), $627-644$.

[MW] C. Moeglin, J.-L. Waldspurger, Sur l'involution de Zelevinski, J. Reine Angew. Math. 372 (1986), $136-177$.

[M] A. Molev, Irreducibility criterion for tensor products of Yangian evaluation modules, Duke Math. J. 112 (2002), 307-341.

[NT1] M. Nazarov, V. Tarasov, On irreducibility of tensor products of Yangian modules, Internat. Math. Res. Notices 3 (1998), 125-150. 
[NT2] M. NAZARov, V. TARASOv, On irreducibility of tensor products of Yangian modules associated with skew Young diagrams, Duke Math. J. 112 (2002), 343-378.

[Re] M. ReIneKe, Multiplicative properties of dual canonical bases of quantum groups, J. Algebra 211 (1999), $134-149$.

[Ro1] J. D. Rogawski, On modules over the Hecke algebra of a p-adic group, Invent. Math. 79 (1985), 443465.

[Ro2] J. D. Rogawski, Representations of $G L(n)$ over a p-adic field with an Iwahori-fixed vector, Israel Math. J. 54 (1986), 242-256.

[Su] T. Suzuki, Rogawski's conjecture on the Jantzen filtration for the degenerate affine Hecke algebra of type A, Elec. J. Represent. Theory 2 (1998), 393-409.

[TT] E. TAFT, J. TowBer, Quantum deformation of flag schemes and Grassman schemes I - A q-deformation of the shape algebra for $G L(n)$, J. of Algebra 142 (1991), 1-36.

[Va] M. Varagnolo, Quiver varieties and Yangians, Lett. Math. Phys. 53 (2000), 273-283.

[Wa] J.-L. WaldSPurger, Algèbres de Hecke et induites de représentations cuspidales pour $G L(N)$, J. Reine Angew. Math. 370 (1986), 127-191.

[Ze1] A. Zelevinsky, Induced representations of reductive p-adic groups II, Ann. Sci. E.N.S. 13 (1980), 165210 .

[Ze2] A. Zelevinsky, A p-adic analog of the Kazhdan-Lusztig conjecture, Funct. Anal. Appl. 15 (1981), 83-92.

[Ze3] A. Zelevinsky, Two remarks on graded nilpotent classes, Russian Math. Surveys 40 (1985), 249-250.

B. LeCLerC: Département de Mathématiques, Université de Caen, Campus II, Bld Maréchal Juin, BP 5186, 14032 Caen cedex, France email : leclerc@math.unicaen.fr

M. NAZARov : Department of Mathematics, University of York, York YO10 5DD, England email : mln1@york.ac.uk

J.-Y. Thibon : Institut Gaspard Monge, Université de Marne-la-Vallée, Champs-sur-Marne, 77454 Marne-la-Vallée cedex 2, France email : jyt@weyl.univ-mlv.fr 